\title{
A new approach to diffeomorphism invariant algebras of generalized functions
}

\author{
E. A. Nigsch
}

July 14, 2018

\begin{abstract}
We develop the diffeomorphism invariant Colombeau-type algebra of nonlinear generalized functions in a modern and compact way. Using a unifying formalism for the local setting and on manifolds, the construction becomes simpler and more accessible than previously in the literature.
\end{abstract}

\section{Introduction}

In the 1980s J. F. Colombeau introduced algebras of nonlinear generalized functions ([3, 4]) in order to overcome the long-standing problem of multiplying distributions, retaining as much compatibility with the classical theory as possible in light of the Schwartz impossibility result ([18]). These algebras and later variations, nowadays simply known as Colombeau algebras, contain the algebra of smooth functions as a faithful subalgebra and the vector space of Schwartz distributions as a linear subspace (see [17, 8] for a comprehensive survey).

A diffeomorphism invariant formulation of the theory was first proposed by Colombeau and Meril in 2], but later seen to be flawed by J. Jelínek who presented a new version in [11, which was subsequently refined in [7]. The difficulties inherent in this development stem from the combination of three facets (see [8, Chapter 2] for a detailed discussion): first, one needs to employ a suitable notion of calculus on (non-Fréchet) locally convex spaces. Second, the proper handling of diffeomorphism invariance manifestly presents a major hurdle in the constructions cited above, both conceptually and technically. And third, establishing stability of the algebra under differentiation is far from trivial and requires a delicate treatment. For this reason the published results in this area consist of several long, technically involved papers which are difficult to assimilate for those not already working in the field.

In this article we give a systematically refined presentation of the global theory of full Colombeau algebras, based on the algebras $\mathcal{G}^{d}$ of $[7$ and $\hat{\mathcal{G}}$ of 9 but replacing a significant part of the preceding foundational material by a succint, more efficient approach.

Our presentation is based, both locally and on manifolds, on the formalism of 9, where so-called smoothing kernels are used as key components of the construction. This not only simplifies the local case in several respects compared to [7] but also makes the translation to manifolds much more convenient. En passant, several proofs of [7] were simplified; in particular, we give a significantly shorter proof of stability under differentiation. Finally, we establish the few core 
properties of smoothing kernels on which the whole theory depends separately, which makes for a clearer and less technical presentation.

\section{Preliminaries}

$B_{r}(x)$ denotes the open ball of radius $r>0$ centered at $x \in \mathbb{R}^{n}$ with respect to the Euclidean metric. $\partial_{i}$ denotes the $i$ th partial derivative; we employ common multi-index notation where for $\alpha=\left(\alpha_{1}, \ldots, \alpha_{n}\right) \in \mathbb{N}_{0}^{n}$ we have $\partial^{\alpha}=\partial_{1}^{\alpha_{1}} \cdots \partial_{n}^{\alpha_{n}} . \partial_{x}^{\alpha}$ means the derivative in the $x$-variable. We abbreviate $\partial_{x+y}^{\alpha}:=\left(\partial_{x}+\partial_{y}\right)^{\alpha}$ which gets expanded by the binomial theorem, $\left(-\partial_{x}\right)^{\alpha}:=$ $(-1)^{|\alpha|} \partial_{x}^{\alpha}$ and $\partial_{(x, y)}^{(\alpha, \beta)}=\partial_{x}^{\alpha} \partial_{y}^{\beta}$. $\mathrm{D}_{X}$ means the directional derivative on functions with respect to a vector field $X$, with $\mathrm{D}_{X}^{x}$ denoting the directional derivative in the variable $x$. $\left\{e_{1}, \ldots, e_{n}\right\}$ is the Euclidean basis of $\mathbb{R}^{n}$.

We use the Landau notation $f(\varepsilon)=O(g(\varepsilon))$ for $\exists \varepsilon_{0}>0, C>0$ : $|f(\varepsilon)| \leq$ $C g(\varepsilon) \forall \varepsilon \leq \varepsilon_{0} . \mathcal{D}(\Omega)$ and $\mathcal{D}^{\prime}(\Omega)$ denote the space of test functions and distributions on $\Omega$, respectively. The action of a distribution $u$ on a test function $\varphi$ is written as $\langle u, \varphi\rangle$. Given open subsets $\Omega, \Omega^{\prime}$ of $\mathbb{R}^{n}$, the pullback $\mu^{*} \rho$ of $\rho \in \mathcal{D}\left(\Omega^{\prime}\right)$ along a diffeomorphism $\mu: \Omega \rightarrow \Omega^{\prime}$ is the element of $\mathcal{D}(\Omega)$ given by $\left(\mu^{*} \rho\right)(y):=\rho(\mu y) \cdot|\operatorname{det} \mathrm{D} \mu(y)|$, where $\mathrm{D} \mu(y)$ is the Jacobian of $\mu$ at $y$ and $\mu_{*}:=\left(\mu^{-1}\right)^{*}$. Accordingly, $\mathrm{L}_{X} \varphi=\mathrm{d} /\left.\mathrm{d} t\right|_{t=0}\left(\left(\alpha_{t}\right)^{*} \varphi\right)$ equals $\mathrm{D}_{X} \varphi+\operatorname{div} X \cdot \varphi$, where $\alpha_{t}$ is the flow of $X$ at time $t$ and $\operatorname{div} X=\sum_{i} \partial X^{i} / \partial x_{i}$. The Lie derivative of a distribution $u$ along $X$ is then given by $\left\langle\mathrm{L}_{X} u, \varphi\right\rangle=-\left\langle u, \mathrm{~L}_{X} \varphi\right\rangle$.

A manifold will always mean an orientable smooth paracompact Hausdorff manifold of finite dimension. The space of distributions on a manifold $M$ is given by $\mathcal{D}^{\prime}(M):=\Omega_{c}^{n}(M)^{\prime}$, where $\Omega^{n}(M)$ is the space of $n$-forms on $M$ and $\Omega_{c}^{n}(M)$ the subspace of those with compact support. We refer to [8, Section 3.1] for a comprehensive exposition of distributions on manifolds. The Lie derivative of functions and $n$-forms on a manifold w.r.t. a vector field $X$ is denoted $\mathrm{L}_{X}$ with $\mathrm{L}_{X}^{x}$ explicitly denoting the derivative in the $x$-variable. $\mathfrak{X}(M)$ is the space of smooth vector fields on $M$ and $B_{r}^{h}(x)$ is the ball of radius $r$ centered at $x$ with respect to a Riemannian metric $h$.

$A \subset \subset B$ means that $A$ is compact and contained in the interior of $B$. We set $I:=(0,1]$. Calculus of smooth functions on infinite-dimensional locally convex vector spaces is to be understood in the sense of convenient calculus of [13, whose basics are presumed to be known. In particular, we use the differentiation operator $d$, the fact that linear bounded maps are smooth, and that the notion of smoothness in convenient calculus agrees with the classical one for finite-dimensional spaces. For a multivariate function $f, \mathrm{~d}_{i} f$ means the differential in the $i$ th variable.

Finally, we refer to [6] for notions of sheaf theory.

\section{Construction of the algebra}

We recall the steps in the construction of a Colombeau algebra on an open set $\Omega \subseteq \mathbb{R}^{n}$. One starts with the basic space $\hat{\mathcal{E}}(\Omega)$, which contains the representatives of generalized functions, together with embeddings of smooth functions and distributions. The action of diffeomorphisms and derivatives on the basic space is then given, extending their classical counterparts. Next follows the 
definition of test objects, which are used to define the subalgebra $\hat{\mathcal{E}}_{m}(\Omega) \subseteq \hat{\mathcal{E}}(\Omega)$ of moderate functions and the ideal $\hat{\mathcal{N}}(\Omega)$ of negligible functions. This in turn gives rise to the quotient algebra $\hat{\mathcal{G}}(\Omega)$. One then verifies the desired properties of the embeddings, the sheaf property and the invariance of negligibility and moderateness under differentiation, which makes the construction complete.

Definition 1. (i) The basic space is $\hat{\mathcal{E}}(\Omega):=C^{\infty}(\mathcal{D}(\Omega) \times \Omega)$, the space of all smooth functions $R:(\varphi, x) \mapsto R(\varphi, x)$ on the product space $\mathcal{D}(\Omega) \times \Omega$. The embeddings $\iota: \mathcal{D}^{\prime}(\Omega) \rightarrow \hat{\mathcal{E}}(\Omega)$ and $\sigma: C^{\infty}(\Omega) \rightarrow \hat{\mathcal{E}}(\Omega)$ are defined as $(\iota u)(\varphi, x)=\langle u, \varphi\rangle$ for a distribution $u$ and $(\sigma f)(\varphi, x)=f(x)$ for a smooth function $f$, where $\varphi \in \mathcal{D}(\Omega)$ and $x \in \Omega$.

(ii) Let $\mu: \Omega \rightarrow \Omega^{\prime}$ be a diffeomorphism onto another open subset $\Omega^{\prime}$ of $\mathbb{R}^{n}$. Given a generalized function $R \in \hat{\mathcal{E}}\left(\Omega^{\prime}\right)$, its pullback $\mu^{*} R \in \hat{\mathcal{E}}(\Omega)$ is defined as $\left(\mu^{*} R\right)(\varphi, x)=R\left(\mu_{*} \varphi, \mu x\right)$.

(iii) The derivative of $R \in \hat{\mathcal{E}}(\Omega)$ with respect to a vector field $X \in C^{\infty}\left(\Omega, \mathbb{R}^{n}\right)$ is defined as $\left(\hat{\mathrm{L}}_{X} R\right)(\varphi, x)=-\mathrm{d}_{1} R(\varphi, x)\left(\mathrm{L}_{X} \varphi\right)+\left(\mathrm{D}_{X}^{x} R\right)(\varphi, x)$.

Remark 2. (i) The formula for $\hat{\mathrm{L}}_{X}$ is obtained by considering the pullback of $R$ along the flow of a (complete) vector field and taking its derivative at time zero.

(ii) One has to verify that $\iota, \sigma, \mu^{*}$ and $\hat{\mathrm{L}}_{X}$ actually map into $\hat{\mathcal{G}}(M)$. First, $\iota u:(\varphi, x) \mapsto \varphi \mapsto\langle u, \varphi\rangle$ is smooth because continuous linear functions are smooth. Second, $\sigma f:(\varphi, x) \mapsto x \mapsto f(x)$ is smooth because $f$ is. Third, $\mu^{*}: \mathcal{D}\left(\Omega^{\prime}\right) \rightarrow \mathcal{D}(\Omega)$ as well as $\mathrm{L}_{X}: \mathcal{D}(\Omega) \rightarrow \mathcal{D}(\Omega)$ are linear and continuous and thus smooth, which implies the same for their extension to $\hat{\mathcal{E}}(\Omega)$.

(iii) $\hat{\mathcal{E}}(\Omega)$ is an associative commutative algebra with unit $\sigma(1):(\varphi, x) \mapsto 1$, $\iota$ is a linear embedding and $\sigma$ an algebra embedding. From the definition one sees that pullback and directional derivatives commute with the embeddings.

(iv) $\hat{\mathrm{L}}_{X}$ is only $\mathbb{R}$-linear but not $C^{\infty}(\Omega)$-linear in $X$; because it commutes with $\iota$, the latter property would in fact give a contradiction similar to the Schwartz impossibility result.

For the quotient construction we employ spaces of smoothing kernels $\widetilde{\mathcal{A}}_{q}(\Omega)$. We give their definition and additional properties now but postpone proofs until Section 7 in order to separate the definitions and main theorems of the theory from the more intricate and technically involved details.

Definition 3. A smoothing kernel of order $q \in \mathbb{N}_{0}$ on an open subset $\Omega$ of $\mathbb{R}^{n}$ is a mapping $\tilde{\phi} \in C^{\infty}(I \times \Omega, \mathcal{D}(\Omega)),(\varepsilon, x) \mapsto\left[y \mapsto \tilde{\phi}_{\varepsilon, x}(y)\right]$, satisfying the following conditions:

(LSK1) $\forall K \subset \subset \Omega \exists \varepsilon_{0}, C>0 \forall x \in K \forall \varepsilon<\varepsilon_{0}: \operatorname{supp} \tilde{\phi}_{\varepsilon, x} \subseteq B_{C \varepsilon}(x)$,

(LSK2) $\forall K \subset \subset \Omega \forall \alpha, \beta \in \mathbb{N}_{0}^{n}:\left(\partial_{x+y}^{\alpha} \partial_{y}^{\beta} \tilde{\phi}\right)_{\varepsilon, x}(y)=O\left(\varepsilon^{-n-|\beta|}\right)$ uniformly for $x \in K$ and $y \in \Omega$,

(LSK3) $\forall K \subset \subset \Omega \forall \alpha \in \mathbb{N}_{0}^{n} \forall f \in C^{\infty}(\Omega): \int_{\Omega} f(y)\left(\partial_{x}^{\alpha} \tilde{\phi}\right)_{\varepsilon, x}(y) \mathrm{d} y=\left(\partial^{\alpha} f\right)(x)+$ $O\left(\varepsilon^{q+1}\right)$ uniformly for $x \in K$. 
The space of all smoothing kernels of order $q$ on $\Omega$ is denoted by $\widetilde{\mathcal{A}}_{q}(\Omega)$ and is an affine subspace of $C^{\infty}(I \times \Omega, \mathcal{D}(\Omega))$. The linear subspace parallel to it, denoted by $\widetilde{\mathcal{A}}_{q 0}(\Omega)$, is given by all $\tilde{\phi}$ satisfying (LSK1), (LSK2) and the following condition:

(LSK3') $\forall K \subset \subset \Omega \forall \alpha \in \mathbb{N}_{0}^{n} \forall f \in C^{\infty}(\Omega): \int_{\Omega} f(y)\left(\partial_{x}^{\alpha} \tilde{\phi}\right)_{\varepsilon, x}(y) \mathrm{d} y=O\left(\varepsilon^{q+1}\right)$ uniformly for $x \in K$.

Remark 4. Given $\tilde{\phi}$ in $\widetilde{\mathcal{A}}_{q}(\Omega)$ or $\widetilde{\mathcal{A}}_{q 0}(\Omega)$ and a vector field $X \in C^{\infty}\left(\Omega, \mathbb{R}^{n}\right)$, $\left(\mathrm{D}_{X}^{x}+\mathrm{L}_{X}^{y}\right) \tilde{\phi}$ is an element of $\widetilde{\mathcal{A}}_{q 0}(\Omega)$. In fact, $\left(\left(\mathrm{D}_{X}^{x}+\mathrm{L}_{X}^{y}\right) \tilde{\phi}\right)_{\varepsilon, x}=\left(\mathrm{D}_{X}^{x+y} \tilde{\phi}\right)_{\varepsilon, x}+$ $\operatorname{div} X \cdot \tilde{\phi}_{\varepsilon, x}$. For (LSK1), let $K \subset \subset \Omega$ and choose $L$ with $K \subset \subset L \subset \subset \Omega$. Then for some $C>0$ such small $\varepsilon, \operatorname{supp} \tilde{\phi}_{\varepsilon, x} \subseteq B_{C \varepsilon}(x) \forall x \in L$, which implies the same for $\left(\mathrm{D}_{X}^{x} \tilde{\phi}\right)_{\varepsilon, x}$ and $\left(\mathrm{D}_{X}^{y} \tilde{\phi}\right)_{\varepsilon, x}$ if $x \in K$. For (LSK2) we note that with $X=\left(X^{1}, \ldots, X^{n}\right),\left(\mathrm{D}_{X}^{x+y} \tilde{\phi}\right)_{\varepsilon, x}(y)$ equals $\sum_{i}\left(\left(X^{i}(x) \partial_{x_{i}+y_{i}}+\left(X^{i}(y)-\right.\right.\right.$ $\left.\left.\left.X^{i}(x)\right) \partial_{y_{i}}\right) \tilde{\phi}\right)_{\varepsilon, x}(y)$; the first term of each summand can be estimated by $O\left(\varepsilon^{-n}\right)$ and the second by

$$
\sup _{y \in B_{C \varepsilon}(x)}\left|X^{i}(y)-X^{i}(x)\right| \cdot \sup _{y \in \Omega}\left|\partial_{y_{i}} \tilde{\phi}_{\varepsilon, x}(y)\right|=O(\varepsilon) O\left(\varepsilon^{-n-1}\right)=O\left(\varepsilon^{-n}\right)
$$

for some $C>0$ uniformly for $x$ in compact sets, and similarly for its derivatives. (LSK3') is clear from the definitions.

Definition 5. Let $\mu: \Omega \rightarrow \Omega^{\prime}$ be a diffeomorphism. We define the pullback $\mu^{*} \tilde{\phi}$ of a smoothing kernel $\tilde{\phi} \in \widetilde{\mathcal{A}}_{q}\left(\Omega^{\prime}\right)$ by $\left(\mu^{*} \tilde{\phi}\right)_{\varepsilon, x}(y):=\mu^{*}\left(\tilde{\phi}_{\varepsilon, \mu x}\right)(y)=\tilde{\phi}_{\varepsilon, \mu x}(\mu y)$. $|\operatorname{det} \mathrm{D} \mu(y)|$.

By smoothness of $\mu$ and $\mu^{*}: \mathcal{D}\left(\Omega^{\prime}\right) \rightarrow \mathcal{D}(\Omega), \mu^{*} \tilde{\phi}=\mu^{*} \circ \tilde{\phi} \circ(\mathrm{id} \times \mu)$ is an element of $C^{\infty}(I \times \Omega, \mathcal{D}(\Omega))$, where id is the identity mapping.

Proposition 6. The smoothing kernels of Definition 3 satisfy these properties:

(LSK4) Let $U, V$ be open subsets of $\Omega, K \subset \subset U \cap V$ and $q \in \mathbb{N}_{0}$. Given $\tilde{\phi} \in \widetilde{\mathcal{A}}_{q}(U)$ there exist $\varepsilon_{0}>0$ and $\tilde{\psi} \in \widetilde{\mathcal{A}}_{q}(V)$ such that $\tilde{\phi}_{\varepsilon, x}=\tilde{\psi}_{\varepsilon, x}$ for $\varepsilon<\varepsilon_{0}$ and $x \in K$.

$(L S K 5) \forall u \in \mathcal{D}^{\prime}(\Omega) \forall \tilde{\phi} \in \widetilde{\mathcal{A}}_{0}(\Omega) \forall k \in \mathbb{N}_{0} \forall X_{1}, \ldots, X_{k} \in C^{\infty}\left(\Omega, \mathbb{R}^{n}\right):\left\langle u, \mathrm{D}_{X_{1}}^{x} \cdots \mathrm{D}_{X_{k}}^{x} \tilde{\phi}_{\varepsilon, x}\right\rangle$ converges to $\mathrm{L}_{X_{1}} \ldots \mathrm{L}_{X_{k}} u$ in $\mathcal{D}^{\prime}(\Omega)$ for $\varepsilon \rightarrow 0$.

(LSK6) Given a diffeomorphism $\mu: \Omega \rightarrow \Omega^{\prime}$ and $\tilde{\phi} \in \widetilde{\mathcal{A}}_{q}\left(\Omega^{\prime}\right), \mu^{*} \tilde{\phi} \in \widetilde{\mathcal{A}}_{q}(\Omega)$.

(LSK 7 Given $\tilde{\phi}_{0} \in \widetilde{\mathcal{A}}_{q}(\Omega), \delta \in \mathbb{N}_{0}^{n}, \tilde{\phi}_{\beta} \in \widetilde{\mathcal{A}}_{q 0}(\Omega)$ for all $\beta \neq 0, \beta \leq \delta$, a sequence $\left(\varepsilon_{j}\right)_{j \in \mathbb{N}}$ with $0<\varepsilon_{j+1}<\varepsilon_{j}<1 / j \forall j \in \mathbb{N}$, a sequence $\left(x_{j}\right)_{j \in \mathbb{N}}$ in a set $K \subset \subset \Omega$ and functions $\lambda_{j}$ as in Lemma 23, the function $\tilde{\psi} \in$ $C^{\infty}(I \times \Omega, \mathcal{D}(\Omega)$ defined by

$$
\tilde{\psi}_{\varepsilon, x}(y):=\sum_{j=1}^{\infty} \lambda_{j}(\varepsilon)\left(\frac{\varepsilon_{j}}{\varepsilon}\right)^{n} \sum_{\beta \leq \delta} \frac{\left(x-x_{j}\right)^{\beta}}{\beta !}\left(\tilde{\phi}_{\beta}\right)_{\varepsilon_{j}, x_{j}}\left(\varepsilon_{j} \frac{y-x}{\varepsilon}+x_{j}\right)
$$

is an element of $\widetilde{\mathcal{A}}_{q}\left(\mathbb{R}^{n}\right)$. 
Remark 7. (LSK4) is of value in several proofs, essentially stating that during testing smoothing kernels can be restricted and extended as needed. In (LSK5) one can equivalently demand that $\left\langle u,\left(\mathrm{D}_{X_{1}}^{x}+\mathrm{L}_{X_{1}}^{y}\right) \cdots\left(\mathrm{D}_{X_{1}}^{x}+\mathrm{L}_{X_{k}}^{y}\right) \tilde{\phi}_{\varepsilon, x}\right\rangle$ converges to 0 for $k>0$ and to zero for $k=0$. (LSK7) gives smoothing kernels taking prescribed values at chosen points and is needed to prove stability of moderateness and negligibility under directional derivatives.

We can now formulate the definitions of moderateness and negligibility.

Definition 8. (i) $R \in \hat{\mathcal{E}}(\Omega)$ is called moderate if $\forall K \subset \subset \Omega \forall \alpha \in \mathbb{N}_{0}^{n} \exists q \in \mathbb{N}_{0}$ $\exists N \in \mathbb{N} \forall \tilde{\phi} \in \widetilde{\mathcal{A}}_{q}(\Omega): \sup _{x \in K}\left|\partial_{x}^{\alpha}\left(R\left(\tilde{\phi}_{\varepsilon, x}, x\right)\right)\right|=O\left(\varepsilon^{-N}\right)$. The set of all moderate elements of $\hat{\mathcal{E}}(\Omega)$ is denoted by $\hat{\mathcal{E}}_{m}(\Omega)$.

(ii) $R \in \hat{\mathcal{E}}(\Omega)$ is called negligible if $\forall K \subset \subset \Omega \forall \alpha \in \mathbb{N}_{0}^{n} \forall m \in \mathbb{N} \exists q \in \mathbb{N}_{0}$ $\forall \tilde{\phi} \in \widetilde{\mathcal{A}}_{q}(\Omega): \sup _{x \in K}\left|\partial_{x}^{\alpha}\left(R\left(\tilde{\phi}_{\varepsilon, x}, x\right)\right)\right|=O\left(\varepsilon^{m}\right)$. The set of all negligible elements of $\hat{\mathcal{E}}(\Omega)$ is denoted by $\hat{\mathcal{N}}(\Omega)$.

Remark 9. In the original definition of $\mathcal{G}^{d}$ the moderateness test (translated to the formalism using smoothing kernels) had to be satisfied for all $\tilde{\phi} \in \widetilde{\mathcal{A}}_{0}(\Omega)$; because this produces a purely technical artefact in the definition of point values and manifold-valued functions $\left(\left[14,[16)\right.\right.$ we prefer the test with $\tilde{\phi} \in \widetilde{\mathcal{A}}_{q}(\Omega)$ for some $q$, where this does not appear. And what's more, this gives in fact an isomorphic algebra, as has been shown in 12. Furthermore, we have stronger conditions on the smoothing kernels than [7], which only requires $\alpha=0$ in (LSK3), but the resulting algebras are again isomorphic ([7, Corollary 16.8]).

As in other variants of the theory the negligibility test is simplified if the tested function is already known to be moderate; the proof uses the same argument as in all the other variants of the theory ([8, Theorem 1.2.3]).

Proposition 10. $R \in \hat{\mathcal{E}}_{m}(\Omega)$ is negligible if and only if Definition 8 (ii) holds for $\alpha=0$, i.e., $\forall K \subset \subset \Omega \forall m \in \mathbb{N} \exists q \in \mathbb{N}_{0} \forall \tilde{\phi} \in \widetilde{\mathcal{A}}_{q}(\Omega): \sup _{x \in K}\left|R\left(\tilde{\phi}_{\varepsilon, x}, x\right)\right|=$ $O\left(\varepsilon^{m}\right)$.

Proof. Suppose $R$ satisfies Definition 8 (ii) for $\alpha=\alpha_{0} \in \mathbb{N}_{0}^{n}$ and fix sets $K_{0} \subset \subset$ $L \subset \subset \Omega, m_{0} \in \mathbb{N}$ and $1 \leq i \leq n$. Testing $R$ for moderateness on $L$ with $\alpha=\alpha_{0}+$ $2 e_{i}$ gives $q_{1} \in \mathbb{N}_{0}$ and $N \in \mathbb{N}$. By assumption the negligibility test on $L$ with $\alpha=$ $\alpha_{0}$ and $m=2 m_{0}+N$ gives some $q_{2} \in \mathbb{N}_{0}$. Take $q=\max \left(q_{1}, q_{2}\right)$ and $\tilde{\phi} \in \widetilde{\mathcal{A}}_{q}(\Omega)$. Define $f_{\varepsilon} \in C^{\infty}(\Omega)$ by $f_{\varepsilon}(x)=\partial_{x}^{\alpha_{0}}\left(R\left(\tilde{\phi}_{\varepsilon, x}, x\right)\right)$. Then for small $\varepsilon, x+[0,1]$. $\varepsilon^{m_{0}+N} e_{i} \subseteq L$ for all $x \in K_{0}$, so $f_{\varepsilon}\left(x+\varepsilon^{m_{0}+N} e_{i}\right)=f_{\varepsilon}(x)+\left(\partial_{x_{i}} f_{\varepsilon}\right)(x) \varepsilon^{m_{0}+N}+$ $\int_{0}^{1}(1-t)\left(\partial_{i}^{2} f_{\varepsilon}\right)\left(x+t \varepsilon^{m_{0}+N} e_{i}\right) \varepsilon^{2 m_{0}+2 N} \mathrm{~d} t$. Then $\left(\partial_{x_{i}} f_{\varepsilon}\right)(x)$ is given by $\left(f_{\varepsilon}(x+\right.$ $\left.\left.\varepsilon^{m_{0}+N} e_{i}\right)-f_{\varepsilon}(x)\right) \cdot \varepsilon^{-m_{0}-N}-\int_{0}^{1}(1-t)\left(\partial_{i}^{2} f_{\varepsilon}\right)\left(x+t \varepsilon^{m_{0}+N} e_{i}\right) \varepsilon^{m_{0}+N} \mathrm{~d} t=O\left(\varepsilon^{m_{0}}\right)$ uniformly for $x \in K_{0}$, which shows that $R$ satisfies the negligibility test on $K_{0}$ for $\alpha=\alpha_{0}+e_{i}$ and $m=m_{0}$. By induction $R$ is negligible.

Theorem 11. (i) $\iota\left(\mathcal{D}^{\prime}(\Omega)\right) \subseteq \hat{\mathcal{E}}_{m}(\Omega)$, (ii) $\sigma\left(C^{\infty}(\Omega)\right) \subseteq \hat{\mathcal{E}}_{m}(\Omega)$, (iii) $(\iota-$ $\sigma)\left(C^{\infty}(\Omega)\right) \subseteq \hat{\mathcal{N}}(\Omega),(i v) \iota\left(\mathcal{D}^{\prime}(\Omega)\right) \cap \hat{\mathcal{N}}(\Omega)=\{0\}$.

Proof. (i) Let $u \in \mathcal{D}^{\prime}(\Omega)$ be given. Fix $K \subset \subset L \subset \subset \Omega, \alpha \in \mathbb{N}_{0}^{n}$ and set $q=0$. Given $\tilde{\phi} \in \widetilde{\mathcal{A}}_{q}(\Omega)$ the moderateness test involves estimating $\partial_{x}^{\alpha}\left((\iota u)\left(\tilde{\phi}_{\varepsilon, x}, x\right)\right)=$ $\partial_{x}^{\alpha}\left\langle u, \tilde{\phi}_{\varepsilon, x}\right\rangle=\left\langle u, \partial_{x}^{\alpha} \tilde{\phi}_{\varepsilon, x}\right\rangle$ for $x \in K$. By (LSK1) $\tilde{\phi}_{\varepsilon, x}$ and its derivatives 
have support in $L$ for small $\varepsilon$ and $x \in K$, so by the usual seminorm estimate for distributions and (LSK2) there exist some $C>0$ and $m \in \mathbb{N}$ depending only on $u$ and $L$ such that this expression can be estimated by $C \sup _{|\beta| \leq m, x \in K, y \in L}\left|\partial_{y}^{\beta} \partial_{x}^{\alpha} \tilde{\phi}_{\varepsilon, x}(y)\right|=O\left(\varepsilon^{-n-|\alpha|-|\beta|}\right)$.

(ii) is clear because derivatives of $f \in C^{\infty}(\Omega)$ are bounded on compact sets independently of $\varepsilon$.

(iii) For $K \subset \subset \Omega, \alpha \in \mathbb{N}_{0}^{n}, f \in C^{\infty}(\Omega)$ and $m \in \mathbb{N}$ we have for all $\tilde{\phi} \in \widetilde{\mathcal{A}}_{m-1}(\Omega)$ that $\partial_{x}^{\alpha}\left((\iota f)\left(\tilde{\phi}_{\varepsilon, x}, x\right)\right)=\left\langle f,\left(\partial_{x}^{\alpha} \tilde{\phi}\right)_{\varepsilon, x}\right\rangle=\left(\partial^{\alpha} f\right)(x)+O\left(\varepsilon^{m}\right)=$ $\partial_{x}^{\alpha}\left((\sigma f)\left(\tilde{\phi}_{\varepsilon, x}, x\right)\right)+O\left(\varepsilon^{m}\right)$ uniformly for $x \in K$ by (LSK3).

(iv) Let $u \in \mathcal{D}^{\prime}(\Omega)$ with $\iota u \in \hat{\mathcal{N}}(\Omega)$ and $\varphi \in \mathcal{D}(\Omega)$. Then with $\tilde{\phi} \in \widetilde{\mathcal{A}}_{q}(\Omega)$ for some $q$ the function in $x$ given by $\left\langle u, \tilde{\phi}_{\varepsilon, x}\right\rangle$ converges to 0 uniformly for $x \in \operatorname{supp} \varphi$ when $\varepsilon \rightarrow 0$ because of negligibility of $\iota u$, thus $\left\langle\left\langle u, \tilde{\phi}_{\varepsilon, x}\right\rangle, \varphi(x)\right\rangle$ converges to 0 . On the other hand, by (LSK5) $\left\langle u, \tilde{\phi}_{\varepsilon, x}\right\rangle$ converges to $u$ in $\mathcal{D}^{\prime}(\Omega)$, which implies $u=0$.

The following is easily verified with the respective definitions.

Theorem 12. $\hat{\mathcal{E}}_{m}(\Omega)$ is a subalgebra of $\hat{\mathcal{E}}(\Omega)$ and $\hat{\mathcal{N}}(\Omega)$ is an ideal in $\hat{\mathcal{E}}_{m}(\Omega)$.

We can now define the algebra of generalized functions on $\Omega$ (isomorphic to $\mathcal{G}^{d}(\Omega)$ of [7]) as the quotient of moderate modulo negligible functions.

Definition 13. $\hat{\mathcal{G}}(\Omega):=\hat{\mathcal{E}}_{m}(\Omega) / \hat{\mathcal{N}}(\Omega)$.

Diffeomorphism invariance of $\hat{\mathcal{G}}$ now follows from (LSK6).

Proposition 14. Let $\mu: \Omega \rightarrow \Omega^{\prime}$ be a diffeomorphism. Then $\mu^{*}\left(\hat{\mathcal{E}}_{m}\left(\Omega^{\prime}\right)\right) \subseteq$ $\hat{\mathcal{E}}_{m}(\Omega)$ and $\mu^{*}\left(\hat{\mathcal{N}}\left(\Omega^{\prime}\right)\right) \subseteq \hat{\mathcal{N}}(\Omega)$, thus $\mu$ is well-defined on $\hat{\mathcal{G}}$ by its action on representatives.

From Remark 2 (iii) it now follows that $\iota$ and $\sigma$, considered as maps into $\hat{\mathcal{G}}(\Omega)$, also commute with diffeomorphisms.

\section{Sheaf properties}

Definition 15. Let $R \in \hat{\mathcal{E}}(\Omega)$ and $\Omega^{\prime} \subseteq \Omega$ open. Then the restriction $\left.R\right|_{\Omega^{\prime}} \in$ $\hat{\mathcal{E}}\left(\Omega^{\prime}\right)$ is defined as $\left.R\right|_{\Omega^{\prime}}(\omega, x):=R(\omega, x)$ for $\omega \in \mathcal{D}\left(\Omega^{\prime}\right) \subseteq \mathcal{D}(\Omega)$ and $x \in \Omega^{\prime}$.

Employing (LSK4) one immediately obtains that moderateness and negligibility are local properties, which makes restriction well-defined also on the quotient space:

Proposition 16. (i) Let $\Omega^{\prime} \subseteq \Omega$ be open and $R \in \hat{\mathcal{E}}(\Omega)$. If $R$ is moderate or negligible, respectively, then so is $\left.R\right|_{\Omega^{\prime}}$.

(ii) Let $\left(U_{\alpha}\right)_{\alpha}$ be an open covering of $\Omega$ and $R \in \hat{\mathcal{E}}(\Omega)$. If for all $\alpha,\left.R\right|_{U_{\alpha}}$ is moderate or negligible, respectively, then so is $R$.

Definition 17. Let $\hat{T} \in \hat{\mathcal{G}}(\Omega)$ and $\Omega^{\prime} \subseteq \Omega$. Then the restriction $\left.\hat{T}\right|_{\Omega^{\prime}} \in \hat{\mathcal{G}}\left(\Omega^{\prime}\right)$ of $\hat{T}$ to $\Omega^{\prime}$ is defined as $\left.\hat{T}\right|_{\Omega^{\prime}}:=\left.T\right|_{\Omega^{\prime}}+\hat{\mathcal{N}}\left(\Omega^{\prime}\right)$ where $T \in \hat{\mathcal{E}}_{m}(\Omega)$ is any representative of $\hat{T}$.

Proposition 18. $\hat{\mathcal{G}}$ is a fine sheaf of differential algebras. 
Proof. Let $U \subseteq \mathbb{R}^{n}$ be open and $\left\{U_{\lambda}\right\}_{\lambda}$ an open cover of $U$. Suppose that for each $\lambda$ we are given an element $\hat{T}_{\lambda} \in \hat{\mathcal{G}}\left(U_{\lambda}\right)$ represented by $T_{\lambda} \in \hat{\mathcal{E}}_{m}\left(U_{\lambda}\right)$ such that $\left.\left(\hat{T}_{\lambda}-\hat{T}_{\mu}\right)\right|_{U_{\lambda} \cap U_{\mu}}$ is zero for all $\lambda$ and $\mu$. We have to show that there exists a generalized function $\hat{T} \in \hat{\mathcal{G}}(U)$ such that $\left.\hat{T}\right|_{U_{\lambda}}=\hat{T}_{\lambda}$ for all $\lambda$. By Proposition 16 (ii), $\hat{T}$ then is unique with this property.

Let $\left\{\chi_{j}\right\}_{j}$ be a locally finite partition of unity such that each $\chi_{j}$ has compact support in $U_{\lambda(j)}$ for some $\lambda(j)$. For each $j$ choose an open neighborhood $W_{j}$ of supp $\chi_{j}$ which is relatively compact in $U_{\lambda(j)}$ and a function $\theta_{j} \in \mathcal{D}\left(U_{\lambda(j)}\right)$ which is 1 on $\overline{W_{j}}$. Define $\pi_{j} \in C^{\infty}\left(\mathcal{D}(U), \mathcal{D}\left(U_{\lambda(j)}\right)\right)$ by $\pi_{j}(\omega):=\theta_{j} \cdot \omega$ for all $j$ and $T \in C^{\infty}(\mathcal{D}(U) \times U)$ by $T(\omega, x):=\sum_{j} \chi_{j}(x) \cdot T_{\lambda(j)}\left(\pi_{j}(\omega), x\right)$. Because the family $\left\{W_{j}\right\}_{j}$ and thus also $\left\{\operatorname{supp} \chi_{j}\right\}_{j}$ are locally finite this sum is well-defined and smooth.

Fix $K \subset \subset U$ and $\alpha \in \mathbb{N}_{0}^{n}$ for the moderateness test. Because $K$ has an open neighborhood intersecting only finitely many supp $\chi_{j}$ there is a finite set $F$ such that for all $\tilde{\phi} \in \widetilde{\mathcal{A}}_{0}(U), \alpha \in \mathbb{N}_{0}^{n}$ and $x \in K, \partial_{x}^{\alpha}\left(T\left(\tilde{\phi}_{\varepsilon, x}, x\right)\right)=\sum_{j \in F} \partial_{x}^{\alpha}\left(\chi_{j}(x)\right.$. $\left.T_{\lambda(j)}\left(\pi_{j}\left(\tilde{\phi}_{\varepsilon, x}\right), x\right)\right)$. For $T$ to be moderate it therefore suffices to show that for each fixed $j \in F$, any $L \subset \subset W_{j}$ and any $\beta \in \mathbb{N}_{0}^{n}$ there exist $q \in \mathbb{N}_{0}$ and $N \in \mathbb{N}$ such that if $\tilde{\phi}$ is of order $q$ then $\partial_{x}^{\beta}\left(T_{\lambda(j)}\left(\pi_{j}\left(\tilde{\phi}_{\varepsilon, x}\right), x\right)\right)=O\left(\varepsilon^{-N}\right)$ uniformly for $x$ in $L$.

Fixing $j, L$ and $\beta$ there are $q$ and $N$ such that for all $\tilde{\psi} \in \widetilde{\mathcal{A}}_{q}\left(U_{\lambda(j)}\right)$ we have $\partial_{x}^{\beta}\left(T_{\lambda(j)}\left(\tilde{\psi}_{\varepsilon, x}, x\right)\right)=O\left(\varepsilon^{-N}\right)$ uniformly for $x \in L$. In particular, given $\tilde{\phi} \in \widetilde{\mathcal{A}}_{q}(U)$ let $\tilde{\psi}$ be determined by (LSK4) such that $\tilde{\psi}_{\varepsilon, x}=\tilde{\phi}_{\varepsilon, x}$ for small $\varepsilon$ and $x$ in an open neighborhood of $L$ whose closure is compact and contained in $W_{j}$. By (LSK1) then for small $\varepsilon, \operatorname{supp} \tilde{\phi}_{\varepsilon, x} \subseteq W_{j}$ for all $x$ in this neighborhood and hence $\partial_{x}^{\beta}\left(T_{\lambda(j)}\left(\pi_{j}\left(\tilde{\phi}_{\varepsilon, x}\right), x\right)\right)=\partial_{x}^{\beta}\left(T_{\lambda(j)}\left(\tilde{\psi}_{\varepsilon, x}, x\right)\right)$ for $x \in L$, which implies moderateness of $T$.

Set $\hat{T}=T+\hat{\mathcal{N}}(U)$. For $\left.\hat{T}\right|_{U_{\lambda}}=\hat{T}_{\lambda}$ it suffices by assumption, Proposition 116 (ii) and because $\left\{W_{k}\right\}_{k}$ is an open cover of $U$, to show negligibility of $\left.T\right|_{U_{\lambda} \cap W_{k}}-\left.T_{\lambda(k)}\right|_{U_{\lambda} \cap W_{k}}$ for all $k$. Because $U_{\lambda} \cap W_{k}$ is relatively compact there is a finite set $F$ such that $\left.\left(T-T_{\lambda(k)}\right)\right|_{U_{\lambda} \cap W_{k}}(\omega, x)$ is given by $\sum_{j \in F} \chi_{j}(x)\left(T_{\lambda(j)}\left(\pi_{j}(\omega), x\right)-T_{\lambda(k)}(\omega, x)\right)$ on its domain of definition. For testing a single summand for negligibility fix $j \in F, K \subset \subset U_{\lambda} \cap W_{k}$ and $m \in \mathbb{N}$. By assumption there exist $q$ and $N$ such that for all $\tilde{\psi} \in \widetilde{\mathcal{A}}_{q}\left(U_{\lambda(j)} \cap U_{\lambda(k)}\right)$, $\left(T_{\lambda(j)}-T_{\lambda(k)}\right)\left(\tilde{\psi}_{\varepsilon, x}, x\right)=O\left(\varepsilon^{m}\right)$ uniformly for $x \in K \cap \operatorname{supp} \chi_{j}$. In particular, given $\tilde{\phi} \in \widetilde{\mathcal{A}}_{q}\left(U_{\lambda} \cap W_{k}\right)$ let $\tilde{\psi}$ be determined by (LSK4) such that $\tilde{\psi}_{\varepsilon, x}=\tilde{\phi}_{\varepsilon, x}$ for $x \in K \cap \operatorname{supp} \chi_{j}$ and small $\varepsilon$. By (LSK1), the support of $\tilde{\phi}_{\varepsilon, x}$ is contained in $W_{j}$ for all $x \in K \cap \operatorname{supp} \chi_{j}$ and small $\varepsilon$. This implies $T_{\lambda(j)}\left(\pi_{j}\left(\tilde{\phi}_{\varepsilon, x}\right), x\right)=T_{\lambda(j)}\left(\tilde{\psi}_{\varepsilon, x}, x\right)$, giving the desired estimate.

That $\hat{\mathcal{G}}$ is fine sheaf may be inferred from the fact that it is a sheaf of modules over the soft sheaf $C^{\infty}$ ([1, Theorem 9.16]).

\section{$5 \quad$ Stability under differentiation}

Theorem 19. Let $R \in \hat{\mathcal{E}}(\Omega)$ and $X \in C^{\infty}\left(\Omega, \mathbb{R}^{n}\right)$. Then $(a) R \in \hat{\mathcal{E}}_{m}(\Omega)$ implies $\hat{\mathrm{L}}_{X} R \in \hat{\mathcal{E}}_{m}(\Omega)$, and (b) $R \in \hat{\mathcal{N}}(\Omega)$ implies $\hat{\mathrm{L}}_{X} R \in \hat{\mathcal{N}}(\Omega)$.

Proof. If $X=e_{i}$ for some $i \in\{1, \ldots, n\}$ set $\kappa:=0$, otherwise assume the result 
holds for $X=e_{i}$ for some $i$ and set $\kappa:=1$. This means that this proof has to be read twice - both cases follow the same scheme, but the second requires the first as a prerequisite. Let $\mu:(t, x) \mapsto \mu_{t} x$ be the flow of $X$. The claim follows from estimates of $\left.\partial_{x}^{\alpha}\left(\partial_{t}-\kappa \mathrm{D}_{X}^{x}\right)\left(R\left(\mu_{-t}^{*} \tilde{\phi}_{\varepsilon, x}, \mu_{t} x\right)\right)\right|_{t=0}$, which by the Mazur-Orlicz polarization formula ([15]) $a_{1} \cdots a_{k}=\frac{1}{k !} \sum_{j=1}^{k}(-1)^{k-j} \sum_{i_{1}<\ldots<i_{j}}\left(a_{i_{1}}+\ldots+\right.$ $\left.a_{i_{j}}\right)^{k}$ (for any $a_{1} \ldots a_{k}$ in a commutative ring) is given by a linear combination of terms $f(t, \varepsilon, x):=\left(\mathrm{D}_{Z}^{x}+c\left(\partial_{t}-\kappa \mathrm{D}_{X}^{x}\right)\right)^{|\alpha|+1}\left(R\left(\mu_{-t}^{*} \tilde{\phi}_{\varepsilon, x}, \mu_{t} x\right)\right)$ at $t=0$ with $Z \in \mathbb{N}_{0}^{n}, Z \leq \alpha, c \in\{0,1\},(Z, c) \neq(0,0)$, for which is hence suffices to verify the growth conditions. Assuming the contrary, $\exists K, \alpha$ (a) $\forall N, q$ (b) $\exists m_{0} \forall q$; $\exists \tilde{\phi} \in \widetilde{\mathcal{A}}_{q}(\Omega) \exists\left(\varepsilon_{j}\right)_{j} \searrow 0, \varepsilon_{j}<1 / j, \exists\left(x_{j}\right)_{j} \in K^{\mathbb{N}}:\left|f\left(0, \varepsilon_{j}, x_{j}\right)\right|>j \cdot \varepsilon_{j}^{-N}$ or $>$ $j \cdot \varepsilon_{j}^{m}$, respectively, $\forall j$. By assumption on $R$ one knows that (a) $\exists q_{0}, N_{0}$ (b) $\exists q_{0}$; $\forall \tilde{\psi} \in \widetilde{\mathcal{A}}_{q_{0}}(\Omega): \sup _{x \in K}\left|\left(\mathrm{D}_{Z}^{x}+c\left(\partial_{x_{i}}-\kappa \partial_{t}\right)\right)^{|\alpha|+1}\left(R\left(\beta_{-t}^{*} \tilde{\psi}_{\varepsilon, x}, \beta_{t} x\right)\right)\right|=O\left(\varepsilon^{-N_{0}}\right)$ or $O\left(\varepsilon^{m}\right)$, respectively, where $\beta$ is the flow of $\kappa e_{i}$. Set $N=N_{0}, q=q_{0}$ above. Using the chain rule ([10]), $f(t, \varepsilon, x)$ is given by

$$
\begin{aligned}
& \sum_{\substack{\pi_{1}, \pi_{2} \\
k_{1}+k_{2}=|\alpha|+1}}\left(\begin{array}{c}
|\alpha|+1 \\
k_{1}
\end{array}\right)\left(\mathrm{d}_{1}^{\left|\pi_{1}\right|} \mathrm{d}_{2}^{\left|\pi_{2}\right|} R\right)\left(\mu_{-t}^{*} \tilde{\phi}_{\varepsilon, x}, \mu_{t} x\right) . \\
& \prod_{B_{1} \in \pi_{1}}\left(\mathrm{D}_{Z}^{x}+c\left(\partial_{t}-\kappa \mathrm{D}_{X}^{x}\right)\right)^{\left|B_{1}\right|}\left(\mu_{-t}^{*} \tilde{\phi}_{\varepsilon, x}\right) \cdot \prod_{B_{2} \in \pi_{2}}\left(\mathrm{D}_{Z}^{x}+c\left(\partial_{t}-\kappa \mathrm{D}_{X}^{x}\right)\right)^{\left|B_{2}\right|}\left(\mu_{t} x\right),
\end{aligned}
$$

where $\pi_{j}$ runs through all partitions of $\left\{1, \ldots, k_{j}\right\},\left|\pi_{j}\right|$ is the number of blocks in $\pi_{j}$, and the products run through all blocks of the respective partition. Applying the chain rule in the same way to $\left(\mathrm{D}_{Z}^{x}+c\left(\partial_{x_{i}}-\kappa \partial_{t}\right)\right)^{|\alpha|+1}\left(R\left(\beta_{-t}^{*} \tilde{\psi}_{\varepsilon, x}, \beta_{t} x\right)\right)$, one sees that this expression is equal to $f(t, \varepsilon, x)$ if $\forall k=0, \ldots,|\alpha|+1$

$$
\begin{aligned}
\left(\mathrm{D}_{Z}^{x}+c\left(\partial_{t}-\kappa \mathrm{D}_{X}^{x}\right)\right)^{k}\left(\mu_{-t}^{*} \tilde{\phi}_{\varepsilon, x}\right) & =\left(\mathrm{D}_{Z}^{x}+c\left(\partial_{x_{i}}+\kappa \partial_{y_{i}}\right)\right)^{k} \tilde{\psi}_{\varepsilon, x} \\
\left(\mathrm{D}_{Z}^{x}+c\left(\partial_{t}-\kappa \mathrm{D}_{X}^{x}\right)\right)^{k}\left(\mu_{t} x\right) & =\left(\mathrm{D}_{Z}^{x}+c\left(\partial_{x_{i}}-\kappa \partial_{t}\right)\right)^{k} \beta_{t} x
\end{aligned}
$$

With $\tilde{\phi}_{\beta}=\partial_{x+y}^{\beta_{i}}\left(\left(\left(Z^{i}+1-c\right) \partial_{x_{i}+y_{i}}-\kappa c\left(\mathrm{D}_{X}^{x}+\mathrm{L}_{X}^{y}\right)\right) /\left(Z^{i}+1\right)\right)^{\beta_{i}} \tilde{\phi}$ for $|\beta| \leq$ $|\alpha|+1$ define $\tilde{\psi}$ as in (LSK7). A short calculation shows that $\left(\partial_{x}^{\gamma-\gamma_{i} e_{i}}\left(Z^{i} \partial_{x_{i}}+\right.\right.$ $\left.\left.c\left(\partial_{x_{i}}+\kappa \partial_{y_{i}}\right)\right)^{\gamma_{i}} \tilde{\psi}\right)_{\varepsilon_{j}, x_{j}}=\left(\partial_{x}^{\gamma-\gamma_{i} e_{i}}\left(Z^{i} \partial_{x_{i}}-c\left(\mathrm{~L}_{X}^{y}+\kappa \mathrm{D}_{X}^{x}\right)\right) \tilde{\phi}_{\varepsilon_{j}, x_{j}}\right.$ and thus (1) holds at $(\varepsilon, x)=\left(\varepsilon_{j}, x_{j}\right) \forall j$ for $|\gamma| \leq k$. Equation (2) holds trivially at $(t, x)=$ $\left(0, x_{0}\right)$ if $\kappa c X\left(x_{0}\right)=0$. Otherwise, by the rectification theorem there is a local diffeomorphism $\rho$ and a vector $v \in \mathbb{R}^{n}$ such that $\mathrm{D} \rho(x) X(x)=v \in \mathbb{R}^{n}$ and $\mu(t, x)=\rho^{-1}(\rho(x)+t v)$ for $(t, x)$ in a neighborhood of $\left(0, x_{0}\right)$, which implies $\left(\left(\mathrm{D}_{X}^{x}\right)^{k} \partial_{t}^{l} \mu\right)(t, x)=d^{k+l}\left(\rho^{-1}\right)(\rho(x)+t v) \cdot v^{k+l}$ and thus $\left(\partial_{t}-\mathrm{D}_{X}^{x}\right)^{k} \mu_{t} x=0=$ $\left(\partial_{x_{i}}-\partial_{t}\right)^{k} \beta_{t} x$. In sum this gives a contradiction to our assumption.

\section{Association}

No discussion of Colombeau algebras would be complete without mention of the concept of association, which provides a means to interpret nonlinear generalized functions in the context of linear distribution theory. We give some elementary results here which are typical for all Colombeau algebras and easily obtained by help of Proposition 6 
Definition 20. $R, S \in \hat{\mathcal{E}}_{m}(\Omega)$ are called associated with each other, written $R \approx S$, if $\forall \psi \in \mathcal{D}(\Omega) \exists q \in \mathbb{N} \forall \tilde{\phi} \in \widetilde{\mathcal{A}}_{q}(\Omega):(R-S)\left(\tilde{\phi}_{\varepsilon, x}, x\right)$ converges, as a function in $x$, to 0 in $\mathcal{D}^{\prime}(\Omega)$ for $\varepsilon \rightarrow 0$.

Because a negligible function evidently is associated with zero this definition is independent of the representatives and we may talk of association of elements of $\hat{\mathcal{G}}(\Omega)$. The following classical results are immediate consequences of (LSK1) and (LSK5):

Proposition 21. (i) For $f \in C^{\infty}(\Omega)$ and $u \in \mathcal{D}^{\prime}(\Omega), \iota(f) \iota(u) \approx \iota(f u)$.

(ii) For $f, g \in C(\Omega), \iota(f) \iota(g) \approx \iota(f g)$.

Proof. (i) $\left\langle f(x)\left\langle u, \tilde{\phi}_{\varepsilon, x}\right\rangle-\left\langle f u, \tilde{\phi}_{\varepsilon, x}\right\rangle, \psi(x)\right\rangle \rightarrow 0$ for all $\tilde{\phi} \in \widetilde{\mathcal{A}}_{0}(\Omega)$ by (LSK5). (ii) For $f, g \in C(\Omega)$ and $\tilde{\phi} \in \widetilde{\mathcal{A}}_{0}(\Omega)$, with $C$ from (LSK1) we can for small $\varepsilon$ estimate the modulus of $\int_{B_{C \varepsilon}(x)} f(y)(g(x)-g(y)) \tilde{\phi}_{\varepsilon, x}(y) \mathrm{d} y$ uniformly for $x$ in compact sets by

$$
\sup _{y \in B_{C \varepsilon}(x)}|f(y)(g(x)-g(y))| \cdot C_{\varepsilon} \cdot \sup _{y \in \Omega}\left|\tilde{\phi}_{\varepsilon, x}(y)\right| \rightarrow 0
$$

where $C_{\varepsilon}=O\left(\varepsilon^{n}\right)$ is the volume of $B_{C \varepsilon}(x)$. In particular this holds for $f=1$, so uniformly on compact sets we have $\left\langle g, \phi_{\varepsilon, x}\right\rangle-g(x) \rightarrow 0$ and boundedness of $\left\langle g, \tilde{\phi}_{\varepsilon, x}\right\rangle$. It follows that for $f, g \in C(\Omega),\left\langle f, \tilde{\phi}_{\varepsilon, x}\right\rangle \cdot\left\langle g, \tilde{\phi}_{\varepsilon, x}\right\rangle-\left\langle f g, \tilde{\phi}_{\varepsilon, x}\right\rangle$, which equals $\left\langle f, \tilde{\phi}_{\varepsilon, x}\right\rangle\left(\left\langle g, \tilde{\phi}_{\varepsilon, x}\right\rangle-g(x)\right)+\left\langle f(y)(g(x)-g(y)), \tilde{\phi}_{\varepsilon, x}(y)\right\rangle$, converges to zero uniformly for $x$ in compact sets and thus weakly in $\mathcal{D}^{\prime}(\Omega)$.

Being associated is a local property:

Lemma 22. Given $R, S \in \hat{\mathcal{E}}_{m}(\Omega)$, if $R$ and $S$ are associated with each other then their restrictions to every open subset of $\Omega$ are so. Conversely, if their restrictions to all elements of an open cover of $\Omega$ are associated with each other, then so are $R$ and $S$.

Proof. The first part is clear using (LSK4): for $U \subseteq \Omega$ open and $\psi \in \mathcal{D}(U)$, Definition 20 gives some $q$ such that for $\tilde{\phi} \in \widetilde{\mathcal{A}}_{q}(\Omega),\left\langle(R-S)\left(\tilde{\phi}_{\varepsilon, x}, x\right), \psi(x)\right\rangle \rightarrow 0$; for any $\tilde{\psi} \in \widetilde{\mathcal{A}}_{q}(U)$ then there exists $\tilde{\phi} \in \widetilde{\mathcal{A}}_{q}(\Omega)$ such that $\tilde{\psi}_{\varepsilon, x}=\tilde{\phi}_{\varepsilon, x}$ for $x \in \operatorname{supp} \psi$ and small $\varepsilon$, thus $\left\langle\left(\left.R\right|_{U}-\left.S\right|_{U}\right)\left(\tilde{\psi}_{\varepsilon, x}, x\right), \psi(x)\right\rangle=\left\langle(R-S)\left(\tilde{\phi}_{\varepsilon, x}, x\right), \psi(x)\right\rangle \rightarrow 0$.

For the second part, let $\psi \in \mathcal{D}(\Omega)$ and an open cover $\left(U_{\alpha}\right)_{\alpha}$ of $\Omega$ be given. Choose a subordinate partition of unity $\left(\chi_{j}\right)_{j}$. With $\psi_{j}:=\chi_{j} \cdot \psi$ we then can write $\psi=\sum \psi_{j}$ for finitely many $j$ which we enumerate as $1,2, \ldots, m$ for some $m \in \mathbb{N}$; furthermore, $\operatorname{supp} \psi_{j} \subseteq U_{\alpha(j)}$ for some $\alpha(j)$.

For each $j=1 \ldots m$ by assumption there exists $q_{j}$ such that for all $\tilde{\phi}_{j} \in$ $\widetilde{\mathcal{A}}_{q_{j}}\left(U_{j}\right),\left\langle\left.(R-S)\right|_{U_{j}}\left(\left(\tilde{\phi}_{j}\right)_{\varepsilon, x}, x\right), \psi_{j}(x)\right\rangle \rightarrow 0$. With $q=\max q_{j}$ and $\tilde{\phi} \in \widetilde{\mathcal{A}}_{q}(\Omega)$, $\left\langle(R-S)\left(\tilde{\phi}_{\varepsilon, x}, x\right), \psi(x)\right\rangle=\sum_{j=1}^{m}\left\langle(R-S)\left(\tilde{\phi}_{\varepsilon, x}, x\right), \psi_{j}(x)\right\rangle$ equals (using (LSK1)) $\sum_{j=1}^{m}\left\langle\left.(R-S)\right|_{U_{i}}\left(\tilde{\phi}_{\varepsilon, x}, x\right), \psi_{j}(x)\right\rangle$.

For each $j$ we can by (LSK4) replace $\tilde{\phi}$ by $\tilde{\phi}_{j} \in \widetilde{\mathcal{A}}_{q}\left(U_{j}\right) \subseteq \widetilde{\mathcal{A}}_{q_{j}}\left(U_{j}\right)$ such that $\tilde{\phi}_{\varepsilon, x}=\left(\tilde{\phi}_{j}\right)_{\varepsilon, x}$ for all $x \in \operatorname{supp} \psi_{j}$ and $\varepsilon \leq \varepsilon_{0}$, from which the claim follows. 


\section{$7 \quad$ Smoothing kernels}

We use the following Lemma ([7, Lemma 10.1]).

Lemma 23. Let $1>\varepsilon_{1}>\varepsilon_{2}>\ldots \rightarrow 0, \varepsilon_{0}=2$. Then there exist $\lambda_{j} \in \mathcal{D}(\mathbb{R})$ $(j=1,2, \ldots)$ having the following properties: 1) $\operatorname{supp} \lambda_{j}=\left[\varepsilon_{j+1}, \varepsilon_{j-1}\right]$, 2) $\lambda_{j}(x)>0$ for $x \in\left(\varepsilon_{j+1}, \varepsilon_{j-1}\right)$, 3) $\sum_{j=1}^{\infty} \lambda_{j}(x)=1$ for $x \in I$, 4) $\lambda_{j}\left(\varepsilon_{j}\right)=1$ and 5) $\lambda_{1}(x)=1$ for $x \in\left[\varepsilon_{1}, 1\right]$.

Proposition 24. $\widetilde{\mathcal{A}}_{q}(\Omega)$ is not empty.

Proof. In case of $\Omega=\mathbb{R}^{n}$ we define the prototypical smoothing kernel $\tilde{\phi}^{\circ} \in$ $C^{\infty}\left(I \times \mathbb{R}^{n}, \mathcal{D}\left(\mathbb{R}^{n}\right)\right)$ by $\tilde{\phi}_{\varepsilon, x}^{\circ}(y):=\varepsilon^{-n} \varphi((y-x) / \varepsilon)$ where $\varphi \in \mathcal{D}\left(\mathbb{R}^{n}\right)$ has integral 1 and vanishing moments of order up to $q$. We verify the conditions of Definition 3 (LSK1) follows from $\operatorname{supp} \varphi((.-x) / \varepsilon)=\varepsilon \operatorname{supp} \varphi+x,($ LSK2) is clear. For (LSK3), $\int f(y)\left(\partial_{x}^{\alpha} \tilde{\phi}^{\circ}\right)_{\varepsilon, x}(y) \mathrm{d} y=\int\left(\partial^{\alpha} f\right)(y) \varepsilon^{-n} \varphi((y-x) / \varepsilon) \mathrm{d} y=$ $\int\left(\partial^{\alpha} f\right)(x+\varepsilon z) \varphi(z) \mathrm{d} z=f^{(\alpha)}(x)+O\left(\varepsilon^{q+1}\right)$ is then obtained by Taylor expansion of $f$ at the point $x$ because $\varphi$ has vanishing moments up to order $q$. Hence, $\tilde{\phi}^{\circ} \in \widetilde{\mathcal{A}}_{q}\left(\mathbb{R}^{n}\right)$.

In the general case of an open subset $\Omega \subseteq \mathbb{R}^{n}$ we choose an increasing sequence $\left(K_{j}\right)_{j \in \mathbb{N}}$ of compact sets $K_{1} \subset \subset K_{2} \subset \subset \ldots$ whose union is $\Omega$ and functions $\chi_{j} \in \mathcal{D}\left(\mathbb{R}^{n}\right)$ such that $\chi_{j} \equiv 1$ on $K_{j}$ and $\operatorname{supp} \chi_{j} \subseteq K_{j+1}$. Let $1>\varepsilon_{1}>\varepsilon_{2}>\ldots \rightarrow 0, \varepsilon_{0}=2$ and choose a partition of unity $\left(\lambda_{j}\right)_{j \in \mathbb{N}}$ on $I$ as in Lemma 23. Define $\tilde{\phi} \in C^{\infty}(I \times \Omega, \mathcal{D}(\Omega))$ by $\tilde{\phi}_{\varepsilon, x}(y):=\sum_{j} \lambda_{j}(\varepsilon) \chi_{j}(y) \tilde{\phi}_{\varepsilon, x}^{\circ}(y)$ for $\varepsilon \in I$ and $x, y \in \Omega$. Then $\tilde{\phi}$ satisfies the conditions of Definition 3 because for each $K \subset \subset \Omega$ the equality $\tilde{\phi}_{\varepsilon, x}=\tilde{\phi}_{\varepsilon, x}^{\circ}$ holds for small $\varepsilon$ and $x \in K$.

For the subsequent proofs we recall the multivariate chain rule from [5] in our notation.

Proposition 25. Let $d, m \in \mathbb{N}, g=\left(g_{1}, \ldots, g_{m}\right): U \subseteq \mathbb{R}^{d} \rightarrow \mathbb{R}^{m}, f: V \subseteq$ $\mathbb{R}^{m} \rightarrow \mathbb{C}$ where $U$ and $V$ are open, and $x_{0} \in U$ be given with $g\left(x_{0}\right) \in V$. Let $0 \neq \alpha \in \mathbb{N}_{0}^{n}$ be given. Assuming $g \in C^{\alpha}(U)$ and $f \in C^{|\alpha|}(V)$,

$$
\partial^{\alpha}(f \circ g)(x)=\sum_{1 \leq|\beta| \leq|\alpha|}\left(\partial^{\beta} f\right)(g(x)) \sum_{p(\alpha, \beta)}(\alpha !) \prod_{j=1}^{|\alpha|} \frac{\left(\partial^{l_{j}} g\right)^{k_{j}}(x)}{k_{j} !\left(l_{j} !\right)^{\left|k_{j}\right|}}
$$

for $x \in U$, where $p(\alpha, \beta)$ consists of all $\left(k_{1}, \ldots, k_{|\alpha|} ; l_{1}, \ldots, l_{|\alpha|}\right) \in\left(\mathbb{N}_{0}^{m}\right)^{|\alpha|} \times$ $\left(\mathbb{N}_{0}^{d}\right)^{|\alpha|}$ such that for some $1 \leq s \leq|\alpha|, k_{i}=0$ and $l_{i}=0$ for $1 \leq i \leq|\alpha|-s$; $\left|k_{i}\right|>0$ for $|\alpha|-s+1 \leq i \leq|\alpha|$; and $0 \prec l_{|\alpha|-s+1} \prec \cdots \prec l_{|\alpha|}$ are such that $\sum_{i=1}^{|\alpha|} k_{i}=\beta, \sum_{i=1}^{|\alpha|}\left|k_{i}\right| l_{i}=\alpha$. Here $\partial^{l_{j}} g=\left(\partial^{l_{j}} g_{1}, \ldots, \partial^{l_{j}} g_{m}\right)$ and $\alpha \prec \beta$ means that either $|\alpha|<|\beta|$ or for some $k<n, \alpha_{i}=\beta_{i}$ for $i \leq k$ and $\alpha_{k+1}<\beta_{k+1}$.

Proposition 26. Given $\tilde{\phi} \in \widetilde{\mathcal{A}}_{q}\left(\Omega^{\prime}\right)$ and a diffeomorphism $\mu: \Omega \rightarrow \Omega^{\prime}, \mu^{*} \tilde{\phi} \in$ $\widetilde{\mathcal{A}}_{q}(\Omega)$.

Proof. We verify the conditions of Definition 3 Set $\tilde{\psi}:=\mu^{*} \tilde{\phi}$. First, (LSK1) follows because $\mu_{\sim}$ is locally Lipschitz continuous. For (LSK2) we have to estimate derivatives of $\tilde{\phi}_{\varepsilon, \mu x}(\mu y) \cdot|\operatorname{det} \mathrm{D} \mu(y)|$. We write $\tilde{\phi}_{\varepsilon}(x, y)=\tilde{\phi}_{\varepsilon, x}(y)$, justified by the 
exponential law [13, 3.12], and define the bijective map $T(x, y):=(x, y-x)$. Because $|\operatorname{det} \mathrm{D} \mu(y)|$ does not depend on $\varepsilon$ and $y$ effectively only ranges over a compact set because of (LSK1), it suffices to estimate derivatives of $\tilde{\phi}_{\varepsilon, \mu x}(\mu y)$; assuming $(\alpha, \beta) \neq(0,0)$ (otherwise the case is trivial) we write $\partial_{x+y}^{\alpha} \partial_{y}^{\beta}\left(\tilde{\phi}_{\varepsilon}(\mu x, \mu y)\right)$ as $\partial_{(x, y)}^{(\alpha, \beta)}\left(\left(\tilde{\phi}_{\varepsilon} \circ T^{-1}\right) \circ\left(T \circ(\mu \times \mu) \circ T^{-1}\right)\right)(T(x, y))$ for $x$ in a compact set $K \subset \subset \Omega$ and $y \in \Omega$. Note that $\tilde{\phi}_{\varepsilon} \circ T^{-1}$ is smooth at $T(\mu(x), \mu(y))$ and $T \circ(\mu \times \mu) \circ T^{-1}$ is smooth at $T(x, y)$. By the chain rule that expression is equal to

$$
\begin{gathered}
\sum_{1 \leq\left|\left(\alpha^{\prime}, \beta^{\prime}\right)\right| \leq|(\alpha, \beta)|}\left(\left(\partial_{(x, y)}^{\left(\alpha^{\prime}, \beta^{\prime}\right)}\left(\tilde{\phi}_{\varepsilon} \circ T^{-1}\right)\right)(T(\mu x, \mu y)) \cdot\right. \\
\left.\sum_{p\left((\alpha, \beta),\left(\alpha^{\prime}, \beta^{\prime}\right)\right)}(\alpha, \beta) ! \prod_{j=1}^{|(\alpha, \beta)|} \frac{\left(\partial^{l_{j}} g\right)^{k_{j}}(T(x, y))}{\left(k_{j} !\right)\left(l_{j} !\right)^{\left|k_{j}\right|}}\right)
\end{gathered}
$$

where $g:=T \circ(\mu \times \mu) \circ T^{-1}$ and $p\left((\alpha, \beta),\left(\alpha^{\prime}, \beta^{\prime}\right)\right)$ consists of tuples $\left(k_{1}, \ldots ; l_{1}, \ldots\right)$ satisfying $\sum k_{i}=\left(\alpha^{\prime}, \beta^{\prime}\right)$ and $\sum\left|k_{i}\right| l_{i}=(\alpha, \beta)$. Noting that

$$
\left(\partial_{(x, y)}^{\left(\alpha^{\prime}, \beta^{\prime}\right)}\left(\tilde{\phi}_{\varepsilon} \circ T^{-1}\right)\right)(T(\mu x, \mu y))=\left(\partial_{x+y}^{\alpha^{\prime}} \partial_{y}^{\beta^{\prime}} \tilde{\phi}\right)_{\varepsilon, \mu x}(\mu y)
$$

we see by (LSK2) that this factor in (4) is $O\left(\varepsilon^{-n-\left|\beta^{\prime}\right|}\right)$. Because $\left|\beta^{\prime}\right|$ can be as large as $|(\alpha, \beta)|$ this growth has to be compensated for by the remaining factors. Now $\left(\partial^{l_{j}} g\right)^{k_{j}}(T(x, y))$ with $l_{j}=\left(l_{j}^{(1)}, l_{j}^{(2)}\right)$ and $k_{j}=\left(k_{j}^{(1)}, k_{j}^{(2)}\right)$ is given by $\left(\right.$ with $\left.0^{0}:=1\right)\left(\partial^{l_{j}^{(1)}} \mu\right)^{k_{j}^{(1)}}(x) \cdot\left(\left(\partial^{l_{j}^{(1)}} \mu\right)(y)-\left(\partial^{l_{j}^{(1)}} \mu\right)(x)\right)^{k_{j}^{(2)}}$ if $l_{j}^{(2)}=0$, and $0^{k_{j}^{(1)}} \cdot\left(\left(\partial^{l_{j}^{(1)}+l_{j}^{(2)}} \mu\right)(y)\right)^{k_{j}^{(2)}}$ if $l_{j}^{(2)} \neq 0$.

From this, (LSK1) and Lipschitz continuity of derivatives of $\mu$ one gains that $\left(\partial^{l_{j}} g\right)^{k_{j}}(T(x, y))$ is $O\left(\varepsilon^{\mid k_{j}^{(2)}} \mid\right)$ if $l_{j}^{(2)}=0$ and $O(1)$ if $l_{j}^{(2)} \neq 0$, so the $\prod_{j}$ in (4) gives $O\left(\varepsilon^{m}\right)$ with $m=\sum_{j}\left|k_{j}^{(2)}\right|-\sum_{j: l_{j}^{(2)} \neq 0}\left|k_{j}^{(2)}\right| \geq\left|\beta^{\prime}\right|-\left|\sum_{j}\right| k_{j}^{(2)}\left|\cdot l_{j}^{(2)}\right| \geq\left|\beta^{\prime}\right|-|\beta|$ which leaves $O\left(\varepsilon^{-n-\beta}\right)$ for the growth of (4) as desired.

For (LSK3), the case of $\alpha=0$ is clear by substitution in the integral. Otherwise, we have by Proposition 25 that $\left(\partial_{x}^{\alpha}\left(\mu^{*} \tilde{\phi}\right)\right)_{\varepsilon, x}(y)=\partial_{x}^{\alpha}\left(\tilde{\phi}_{\varepsilon, \mu x}(\mu y)\right.$. $|\operatorname{det} \mathrm{D} \mu(y)|)$ is given by

$$
\sum_{1 \leq|\beta| \leq|\alpha|}\left(\partial_{x}^{\beta} \tilde{\phi}\right)_{\varepsilon, \mu x}(\mu y) \cdot|\operatorname{det} \mathrm{D} \mu(y)| \sum_{p(\alpha, \beta)} \alpha ! \prod_{j=1}^{|\alpha|} \frac{\left(\partial^{l_{j}} \mu\right)^{k_{j}}(x)}{k_{j} !\left(l_{j} !\right)^{\left|k_{j}\right|}}
$$

where $p(\alpha, \beta)=\left(k_{1}, \ldots, k_{|\alpha|} ; l_{1}, \ldots, l_{|\alpha|}\right)$. When integrating the product of this with $f(y)$, substitution gives

$$
\begin{aligned}
& \sum_{1 \leq|\beta| \leq|\alpha|} \int_{\Omega} f(y)\left(\partial_{x}^{\beta} \tilde{\phi}\right)_{\varepsilon, \mu x}(\mu y)|\operatorname{det} \mathrm{D} \mu(y)| \mathrm{d} y \cdot \sum_{p(\alpha, \beta)} \alpha ! \prod_{j=1}^{|\alpha|} \frac{\left(\partial^{l_{j}} \mu\right)^{k_{j}}(x)}{k_{j} !\left(l_{j} !\right)^{\left|k_{j}\right|}} \\
= & \sum_{1 \leq|\beta| \leq|\alpha|} \underbrace{\int\left(f \circ \mu^{-1}\right)(y)\left(\partial_{x}^{\beta} \tilde{\phi}\right)_{\varepsilon, \mu x}(y) \mathrm{d} y}_{=\partial_{x}^{\beta}\left(f \circ \mu^{-1}\right)(\mu(x))+O\left(\varepsilon^{q+1}\right)} \cdot \sum_{p\left(\alpha, \alpha^{\prime}\right)} \alpha ! \prod_{j=1}^{|\alpha|} \frac{\left(\partial^{l_{j}} \mu\right)^{k_{j}}(x)}{k_{j} !\left(l_{j} !\right)^{\left|k_{j}\right|}} \\
= & \left(\left(f \circ \mu^{-1}\right) \circ \mu\right)^{(\alpha)}(x)+O\left(\varepsilon^{q+1}\right)=\left(\partial^{\alpha} f\right)(x)+O\left(\varepsilon^{q+1}\right)
\end{aligned}
$$

uniformly for $x$ in compact sets, which is the desired result. 
We will now show (LSK1-D) for the smoothing kernels of Definition 3 and thus establish Proposition 6 .

Proof of Proposition [6. (LSK4): Let $U, V$ be open subsets of $\Omega, K \subset \subset U \cap V$, $q \in \mathbb{N}_{0}$ and $\tilde{\phi} \in \widetilde{\mathcal{A}}_{q}(U)$. Choose $\chi \in \mathcal{D}(U \cap V)$ with $\chi \equiv 1$ on $K$. Let $\varepsilon_{0} \in I$ be such that $\operatorname{supp} \tilde{\psi}_{\varepsilon, x} \subseteq U \cap V$ for $x \in \operatorname{supp} \chi$ and $\varepsilon \leq \varepsilon_{0}$ and fix any $\lambda \in C^{\infty}(I)$ which is 1 on $\left(0, \varepsilon_{0} / 2\right)$ and 0 on $\left[\varepsilon_{0}, 1\right]$. Fix an arbitary smoothing kernel $\tilde{\psi}^{\circ} \in \widetilde{\mathcal{A}}_{q}(V)$ and define $\tilde{\psi}_{\varepsilon, x}:=\lambda(\varepsilon) \chi(x) \tilde{\phi}_{\varepsilon, x}+(1-\lambda(\varepsilon) \chi(x)) \tilde{\psi}_{\varepsilon, x}^{\circ}$. Then $\tilde{\psi} \in \widetilde{\mathcal{A}}_{q}(V)$ : any given $L \subset \subset V$ can be decomposed as $L=L_{1} \cup L_{2}$ with $L_{1} \subset \subset U \cap V$ and $L_{2} \subset \subset V \backslash \operatorname{supp} \chi$; for $\varepsilon \leq \varepsilon_{0} / 2$, (LSK1), (LSK2) and (LSK3) then are easily seen to be satisfied on $L_{1}$ and $L_{2}$. For $\varepsilon \leq \varepsilon_{0} / 2$ and $x \in K$, finally, $\tilde{\psi}_{\varepsilon, x}=\tilde{\phi}_{\varepsilon, x}$.

(LSK5): Let $u \in \mathcal{D}^{\prime}(\Omega), k \in \mathbb{N}_{0}, X_{1}, \ldots, X_{k} \in C^{\infty}\left(\Omega, \mathbb{R}^{n}\right)$ and $\varphi \in \mathcal{D}(\Omega)$. By (LSK1) supp $\tilde{\phi}_{\varepsilon, x}$ is contained, for small $\varepsilon$, in a relatively compact open neighborhood $U$ in $\Omega$ of $\operatorname{supp} \varphi$ for all $x \in \operatorname{supp} \varphi$. By the structure theorem for distributions we can write $\left.u\right|_{U}=\left.(-1)^{|\beta|} \partial^{\beta} f\right|_{U}$ for a continuous function $f$ with support in an arbitrarily small neighborhood of $\bar{U}$, so $\left\langle\left\langle u, \mathrm{~L}_{X_{1}}^{x} \ldots \mathrm{L}_{X_{k}}^{x} \tilde{\phi}_{\varepsilon, x}\right\rangle, \varphi(x)\right\rangle$ is given by

$$
\begin{aligned}
& \left\langle\left\langle f(y),\left(\partial_{y}^{\beta} \tilde{\phi}\right)_{\varepsilon, x}(y)\right\rangle,(-1)^{k} \mathrm{~L}_{X_{1}} \ldots \mathrm{L}_{X_{k}} \varphi(x)\right\rangle \\
& =\left\langle\left\langle f(y),\left(\left(\partial_{x+y}-\partial_{x}\right)^{\beta} \tilde{\phi}\right)_{\varepsilon, x}(y)\right\rangle,(-1)^{k} \mathrm{~L}_{X_{1}} \ldots \mathrm{L}_{X_{k}} \varphi(x)\right\rangle \\
& =\sum_{\beta^{\prime} \leq \beta}\left(\begin{array}{c}
\beta \\
\beta^{\prime}
\end{array}\right)\left\langle\left\langle f(y),\left(\partial_{x+y}^{\beta^{\prime}}\left(-\partial_{x}\right)^{\beta-\beta^{\prime}} \tilde{\phi}\right)_{\varepsilon, x}(y)\right\rangle,(-1)^{k} \mathrm{~L}_{X_{1}} \ldots \mathrm{L}_{X_{k}} \varphi(x)\right\rangle \\
& =\sum_{\beta^{\prime} \leq \beta}\left(\begin{array}{c}
\beta \\
\beta^{\prime}
\end{array}\right)\left\langle\left\langle f(y),\left(\partial_{x+y}^{\beta^{\prime}} \tilde{\phi}\right)_{\varepsilon, x}(y)\right\rangle,(-1)^{k} \partial^{\beta-\beta^{\prime}} \mathrm{L}_{X_{1}} \ldots \mathrm{L}_{X_{k}} \varphi(x)\right\rangle \\
& =\sum_{\beta^{\prime} \leq \beta}\left(\begin{array}{c}
\beta \\
\beta^{\prime}
\end{array}\right)\left(\left\langle\left\langle f(y)-f(x),\left(\partial_{x+y}^{\beta^{\prime}} \tilde{\phi}\right)_{\varepsilon, x}(y)\right\rangle,(-1)^{k} \partial^{\beta-\beta^{\prime}} \mathrm{L}_{X_{1}} \ldots \mathrm{L}_{X_{k}} \varphi(x)\right\rangle\right. \\
& \left.+\left\langle f(x)\left\langle 1,\left(\partial_{x+y}^{\beta^{\prime}} \tilde{\phi}\right)_{\varepsilon, x}(y)\right\rangle,(-1)^{k} \partial^{\beta-\beta^{\prime}} \mathrm{L}_{X_{1}} \ldots \mathrm{L}_{X_{k}} \varphi(x)\right\rangle\right)
\end{aligned}
$$

Because $f(y)-f(x) \rightarrow 0$ uniformly for $x \in \operatorname{supp} \varphi, y \in B_{C \varepsilon(x)}$ (with $C$ from (LSK1)) and $\varepsilon \rightarrow 0$ and because $\partial_{x+y}^{\beta^{\prime}} \tilde{\phi}_{\varepsilon, x}(y)$ is bounded as in (LSK2) the first part of the last sum converges to 0 similarly as in (3). By (LSK3) the limit of the second part is $\left\langle f(x),(-1)^{k} \partial^{\beta} \mathrm{L}_{X_{1}} \ldots \mathrm{L}_{X_{k}} \varphi(x)\right\rangle=\left\langle\mathrm{L}_{X_{1}} \ldots \mathrm{L}_{X_{k}} u, \varphi\right\rangle$.

(LSK6) was shown in Proposition 26.

(LSK7): (LSK1) for $\tilde{\psi}$ is obvious.

(LSK2) for $\tilde{\psi}$ : For $\alpha \leq \delta$ (otherwise the expression is 0 ) the derivative $\left(\partial_{x+y}^{\alpha} \partial_{y}^{\beta} \tilde{\psi}\right)_{\varepsilon, x}(y)$ is given by

$$
\sum_{j=1}^{\infty} \lambda_{j}(\varepsilon) \sum_{\alpha \leq \delta^{\prime} \leq \delta}\left(\frac{\left(x-x_{j}\right)^{\delta^{\prime}-\alpha}}{\left(\delta^{\prime}-\alpha\right) !}\left(\frac{\varepsilon_{j}}{\varepsilon}\right)^{n+|\beta|} \partial_{y}^{\beta}\left(\tilde{\phi}_{\delta^{\prime}}\right)_{\varepsilon_{j}, x_{j}}\left(\varepsilon_{j} \frac{y-x}{\varepsilon}+x_{j}\right)\right)
$$

By (LSK2) this can be estimated uniformly for $x \in K$ by $\sum_{j} \lambda_{j}(\varepsilon) C\left(\varepsilon_{j} / \varepsilon\right)^{n+|\beta|} \varepsilon_{j}^{-n-|\beta|}=$ $\sum_{j} \lambda_{j}(\varepsilon) C \varepsilon^{-n-|\beta|}=O\left(\varepsilon^{-n-|\beta|}\right)$ for some constant $C>0$.

(LSK3) for $\tilde{\psi}$ is equivalent to $\int f(y)\left(\partial_{x+y}^{\alpha} \tilde{\psi}\right)_{\varepsilon, x}(y) \mathrm{d} y=\partial^{\alpha}(f(x))+O\left(\varepsilon^{q+1}\right)$ for $\alpha \leq \delta$. Note that $\partial^{\alpha}(f(x))$ means the derivative of the constant $f(x)$, which 
is zero for $\alpha \neq 0$. The integral is (for $\varepsilon \leq \varepsilon_{0}$ with $C, \varepsilon_{0}$ from (LSK1))

$$
\sum_{j=1}^{\infty} \lambda_{j}(\varepsilon) \sum_{\alpha \leq \delta^{\prime} \leq \delta} \frac{\left(x-x_{j}\right)^{\delta^{\prime}-\alpha}}{\left(\delta^{\prime}-\alpha\right) !} \int_{B_{C \varepsilon}(x)} f(y)\left(\tilde{\phi}_{\delta^{\prime}}\right)_{\varepsilon_{j}, x_{j}}\left(\varepsilon_{j} \frac{y-x}{\varepsilon}+x_{j}\right) \mathrm{d} y .
$$

Substituting $u=\varepsilon_{j}(y-x) / \varepsilon+x_{j}$ and forming the Taylor expansion of $f(\varepsilon(u-$ $\left.\left.x_{j}\right) / \varepsilon_{j}+x\right)$ of order $q$ about $x, \int f(y)\left(\partial_{x+y}^{\alpha} \tilde{\psi}\right)_{\varepsilon, x}(y) \mathrm{d} y-\partial^{\alpha}(f(x))$ without the remainder term is given by

$$
\begin{aligned}
\sum_{j=1}^{\infty} \sum_{\alpha \leq \delta^{\prime} \leq \delta} \sum_{|\gamma| \leq q} \lambda_{j}(\varepsilon) \frac{\left(x-x_{j}\right)^{\delta^{\prime}-\alpha}}{\left(\delta^{\prime}-\alpha\right) !}\left(\frac{\varepsilon_{j}}{\varepsilon}\right)^{-|\gamma|} \frac{f^{(\gamma)}(x)}{\gamma !} . \\
\quad\left(\int_{B_{C \varepsilon_{j}}\left(x_{j}\right)}\left(u-x_{j}\right)^{\gamma}\left(\tilde{\phi}_{\delta^{\prime}}\right)_{\varepsilon_{j}, x_{j}}(u) \mathrm{d} u-\partial^{\gamma+\delta^{\prime}} 1\right) .
\end{aligned}
$$

The term in parantheses is $O\left(\varepsilon_{j}^{q+1}\right)$ so (5) can be estimated uniformly for $x \in K$ by $\sum_{j=1}^{\infty} \sum_{|\gamma| \leq q} \lambda_{j}(\varepsilon)\left(\varepsilon_{j} / \varepsilon\right)^{-|\gamma|} O\left(\varepsilon_{j}^{q+1}\right)=O\left(\varepsilon^{q+1}\right)$. The remainder is

$$
\begin{aligned}
& \sum_{j=1}^{\infty} \sum_{\alpha \leq \delta^{\prime} \leq \delta} \sum_{|\gamma|=q+1} \lambda_{j}(\varepsilon) \frac{\left(x-x_{j}\right)^{\delta^{\prime}-\alpha}}{\left(\delta^{\prime}-\alpha\right) !} \frac{q+1}{\gamma !} \varepsilon^{q+1} . \\
& \int_{B_{C \varepsilon_{j}}\left(x_{j}\right)} \int_{0}^{1}(1-s)^{q}\left(\partial^{\gamma} f\right)\left(x+s \varepsilon\left(u-x_{j}\right) / \varepsilon_{j}\right) \mathrm{d} s\left(\frac{u-x_{j}}{\varepsilon_{j}}\right)^{\gamma}\left(\tilde{\phi}_{\delta^{\prime}}\right)_{\varepsilon_{j}, x_{j}}(u) \mathrm{d} u .
\end{aligned}
$$

The double integral is bounded uniformly for $x \in K$, so $O\left(\varepsilon^{q+1}\right)$ remains.

\section{Global Theory}

We will now extend the construction to manifolds. This requires little more than the right definitions, with which all properties follow effortlessly from the local case.

Definition 27. Let $M$ be a manifold.

(i) The basic space is $\hat{\mathcal{E}}(M):=C^{\infty}\left(\Omega_{c}^{n}(M) \times M\right)$. The embeddings $\iota: \mathcal{D}^{\prime}(M) \rightarrow$ $\hat{\mathcal{E}}(M)$ and $\sigma: C^{\infty}(M) \rightarrow \hat{\mathcal{E}}(M)$ are defined as $(\iota u)(\omega, x)=\langle u, \omega\rangle$ for a distribution $u$ and $(\sigma f)(\omega, x)=f(x)$ for a smooth function $f$ on $M$, where $\omega \in \Omega_{c}^{n}(M)$ and $x \in M$.

(ii) Let $\mu: M \rightarrow M^{\prime}$ be a diffeomorphism from $M$ to another manifold $M^{\prime}$. Given a generalized function $R \in \hat{\mathcal{E}}\left(M^{\prime}\right)$, its pullback $\mu^{*} R \in \hat{\mathcal{E}}(M)$ is defined as $\left(\mu^{*} R\right)(\omega, x)=R\left(\mu_{*} \omega, \mu x\right)$.

(iii) The Lie derivative of $R \in \hat{\mathcal{E}}(M)$ with respect to a smooth vector field $X$ on $M$ is defined as $\left(\hat{\mathrm{L}}_{X} R\right)(\omega, x)=-\mathrm{d}_{1} R(\omega, x)\left(\mathrm{L}_{X} \omega\right)+\left(\mathrm{L}_{X}^{x} R\right)(\omega, x)$.

Remark 28. By the same reasoning as in the local case $\mu^{*} R$ and $\hat{\mathrm{L}}_{X} R$ are smooth; $\hat{\mathcal{E}}(M)$ is an associative, commutative algebra with unit $\sigma(1):(\omega, x) \mapsto$ $1, \iota$ is a linear embedding and $\sigma$ an algebra embedding. As before, pullback and Lie derivatives commute with the embeddings and $\hat{\mathrm{L}}_{X}$ is only $\mathbb{R}$-linear in $X$ but not $C^{\infty}(M)$-linear. 
We use the following notation for the relationship between local and global expressions on a chart $(U, \psi)$ :

(i) For smooth vector fields, the isomorphism $\mathfrak{X}(U) \cong C^{\infty}\left(\psi(U), \mathbb{R}^{n}\right)$ is written as $X \mapsto X_{U}$ with inverse $Y \mapsto Y^{U}$.

(ii) For $n$-forms, the isomorphism $\Omega^{n}(U) \cong C^{\infty}(\psi(U))$ is written as $\omega \mapsto \omega_{U}$ with inverse $\varphi \mapsto \varphi^{U}$, where $\omega_{U}(y):=\varphi_{*}(\omega)(y)\left(e_{1}, \ldots, e_{n}\right)$.

(iii) For distributions, the isomorphism $\mathcal{D}^{\prime}(U) \cong \mathcal{D}^{\prime}(\varphi(U))$ is given by $u \mapsto u_{U}$ with $\left\langle u_{U}, \varphi\right\rangle:=\left\langle u, \varphi^{U}\right\rangle$ and its inverse $v \mapsto v^{U},\left\langle v^{U}, \omega\right\rangle:=\left\langle v, \omega_{U}\right\rangle$.

(iv) The isomorphism of basic spaces $C^{\infty}\left(\Omega_{c}^{n}(U) \times U\right) \cong C^{\infty}(\mathcal{D}(\varphi(U)) \times \varphi(U))$ is given by $R \mapsto R_{U}$ with $R_{U}(\varphi, x):=R\left(\varphi^{U}, \varphi^{-1} x\right)$ with inverse $S \mapsto S^{U}$, $S^{U}(\omega, x):=S\left(\omega_{U}, \varphi x\right)$.

We then have $\left(\mathrm{L}_{X} \omega\right)_{U}=\mathrm{L}_{X_{U}}\left(\omega_{U}\right)$ and $\left(\hat{\mathrm{L}}_{X} R\right)(\omega, x)=\left(\hat{\mathrm{L}}_{X_{U}} R_{U}\right)\left(\omega_{U}, \varphi x\right)$. Next we define smoothing kernels on manifolds.

Definition 29. A smoothing kernel of order $q \in \mathbb{N}_{0}$ on a manifold $M$ is defined to be a mapping $\Phi \in C^{\infty}\left(I \times M, \Omega_{c}^{n}(M)\right),(\varepsilon, x) \rightarrow\left[y \rightarrow \Phi_{\varepsilon, x}(y)\right]$, satisfying the following conditions for any Riemannian metric $h$ on $M$ :

(SK1) $\forall K \subset \subset M \exists \varepsilon_{0}, C>0 \forall x \in K \forall \varepsilon<\varepsilon_{0}: \operatorname{supp} \Phi_{\varepsilon, x} \subseteq B_{C \varepsilon}^{h}(x)$,

(SK2) $\forall K \subset \subset M \forall j, k \in \mathbb{N}_{0} \forall X_{1}, \ldots, X_{j}, Y_{1}, \ldots, Y_{k} \in \mathfrak{X}(M)$ :

$$
\left\|\left(\mathrm{L}_{X_{1}}^{x+y} \cdots \mathrm{L}_{X_{j}}^{x+y} \mathrm{~L}_{Y_{1}}^{y} \cdots \mathrm{L}_{Y_{k}}^{y} \Phi\right)_{\varepsilon, x}(y)\right\|_{h}=O\left(\varepsilon^{-n-k}\right)
$$

uniformly for $x \in K$ and $y \in M$,

(SK3) $\forall K \subset \subset M \forall j \in \mathbb{N}_{0} \forall X_{1}, \ldots, X_{j} \in \mathfrak{X}(M) \forall f \in C^{\infty}(M)$ :

$$
\int_{M} f \cdot\left(\mathrm{L}_{X_{1}}^{x} \cdots \mathrm{L}_{X_{j}}^{x} \Phi\right)_{\varepsilon, x}=\left(\mathrm{L}_{X_{1}} \cdots \mathrm{L}_{X_{j}} f\right)(x)+O\left(\varepsilon^{q+1}\right)
$$

uniformly for $x \in K$.

The space of all smoothing kernels of order $q$ on $M$ is denoted by $\widetilde{\mathcal{A}}_{q}(M)$ and is an affine subspace of $C^{\infty}\left(I \times M, \Omega_{c}^{n}(M)\right)$. The linear subspace parallel to it, denoted by $\widetilde{\mathcal{A}}_{q 0}(M)$, is given by all $\Phi$ satisfying (SK1), (SK2) and the following condition:

(SK3') $\forall K \subset \subset M \forall j \in \mathbb{N}_{0} \forall X_{1}, \ldots, X_{j} \in \mathfrak{X}(M) \forall f \in C^{\infty}(M)$ :

$$
\int_{M} f \cdot\left(\mathrm{L}_{X_{1}}^{x} \cdots \mathrm{L}_{X_{j}}^{x} \Phi\right)_{\varepsilon, x}=O\left(\varepsilon^{q+1}\right)
$$

uniformly for $x \in K$.

Note that by [9, Lemma 3.4] Definition 29 does not depend on the choice of the Riemannian metric. Given a chart $(U, \varphi)$ on $M$ we see that smoothing kernels on $U$ correspond exactly to smoothing kernels on $\varphi(U)$ as in Definition 3 
Proposition 30. Let $(U, \varphi)$ be a chart on $M$. Then $\widetilde{\mathcal{A}}_{q}(U) \cong \widetilde{\mathcal{A}}_{q}(\varphi(U))$ as affine spaces and $\widetilde{\mathcal{A}}_{q 0}(U) \cong \widetilde{\mathcal{A}}_{q 0}(\varphi(U))$ as linear spaces.

Proof. The isomorphism is $\tilde{\phi}_{\varepsilon, x}:=\left(\Phi_{\varepsilon, \varphi^{-1} x}\right)_{U}$ with inverse $\Phi_{\varepsilon, x}:=\left(\tilde{\phi}_{\varepsilon, \varphi x}\right)^{U}$. Taking for $h$ the pullback metric of the Euclidean metric on $\varphi(U)$ to $U$ along $\varphi$, then given $K \subset \subset \varphi(U) \exists \varepsilon_{0}, C$ such that $\operatorname{supp} \Phi_{\varepsilon, x} \subseteq B_{C \varepsilon}^{h}(x) \forall \varepsilon \leq \varepsilon_{0} \forall x \in$ $\varphi^{-1}(K)$ and thus $\operatorname{supp} \tilde{\phi}_{\varepsilon, x}=\varphi\left(\operatorname{supp} \Phi_{\varepsilon, \varphi^{-1} x}\right) \subseteq \varphi\left(B_{C \varepsilon}^{h}\left(\varphi^{-1}(x)\right)\right)=B_{C \varepsilon}(x)$ $\forall \varepsilon \leq \varepsilon_{0}, x \in K$, thus (SK1) implies (LSK1) for $\tilde{\phi}$; the converse holds by the same reasoning.

Then, $\left(\partial_{i_{1}}^{x+y} \cdots \partial_{i_{k}}^{x+y} \partial_{j_{1}}^{y} \cdots \partial_{j_{l}}^{y} \tilde{\phi}\right)_{\varepsilon, x}$ equals $\left(\left(\mathrm{L}_{\partial_{i_{1}}}^{x+y} \cdots \mathrm{L}_{\partial_{i_{k}}}^{x+y} \mathrm{~L}_{\partial_{j_{1}}}^{y} \cdots \mathrm{L}_{\partial_{j_{l}}}^{y} \Phi\right)_{\varepsilon, \varphi^{-1} x}\right)_{U}$ which implies that (LSK2) for $\tilde{\phi}$ is equivalent to (SK2) for $\Phi$, because in (SK2) it obviously suffices to restrict the $X_{1}, \ldots, Y_{k}$ to be elements of $\left\{\partial_{1}, \ldots, \partial_{n}\right\}$.

By the same reasoning, (LSK3) for $\tilde{\phi}$ is equivalent to (SK3) for $\Phi$ because of

$$
\int_{U} f \cdot\left(\mathrm{L}_{\partial_{i_{1}}}^{x} \cdots \mathrm{L}_{\partial_{i_{j}}}^{x} \Phi\right)_{\varepsilon, x}=\int_{\varphi(U)}\left(f \circ \varphi^{-1}\right)(y) \cdot\left(\partial_{i_{1}}^{x} \cdots \partial_{i_{k}}^{x} \tilde{\phi}\right)_{\varepsilon, \varphi x}(y) \mathrm{d} y
$$

and similarly for (LSK3') and (SK3').

Using this isomorphism we also write $\tilde{\phi}=\Phi_{U}$ and $\Phi=\tilde{\phi}^{U}$, respectively.

Definition 31. Let $\mu: M \rightarrow M^{\prime}$ be a diffeomorphism. Then we define the pullback $\mu^{*} \Phi$ of a smoothing kernel $\Phi \in \widetilde{\mathcal{A}}_{q}\left(M^{\prime}\right)$ by $\left(\mu^{*} \Phi\right)_{\varepsilon, x}:=\mu^{*}\left(\Phi_{\varepsilon, \mu x}\right)$.

Proposition 32. The smoothing kernels of Definition 29 satisfy these additional properties:

(SK4) Let $U, V$ be open subsets of $M, K \subset \subset U \cap V$ and $q \in \mathbb{N}_{0}$. Given $\Phi \in \widetilde{\mathcal{A}}_{q}(U)$ there exist $\varepsilon_{0}>0$ and $\Psi \in \widetilde{\mathcal{A}}_{q}(V)$ such that $\Phi_{\varepsilon, x}=\Psi_{\varepsilon, x}$ for $\varepsilon<\varepsilon_{0}$ and $x \in K$.

(SK5) $\forall u \in \mathcal{D}^{\prime}(M) \forall \Phi \in \widetilde{\mathcal{A}}_{0}(M) \forall k \in \mathbb{N}_{0} \forall X_{1}, \ldots, X_{k} \in \mathfrak{X}(M):\left\langle u, \mathrm{~L}_{X_{1}}^{x} \ldots \mathrm{L}_{X_{k}}^{x} \Phi_{\varepsilon, x}\right\rangle$ converges (weakly) to $\mathrm{L}_{X_{1}} \ldots \mathrm{L}_{X_{k}} u$ in $\mathcal{D}^{\prime}(M)$.

(SK6) If $\mu: M \rightarrow M^{\prime}$ is a diffeomorphism and $\Phi^{\prime} \in \widetilde{\mathcal{A}}_{q}\left(M^{\prime}\right)$ then $\mu^{*} \Phi^{\prime} \in \widetilde{\mathcal{A}}_{q}(M)$.

Proof. (SK4) is proven exactly as in the local case.

(SK5): Let $\omega \in \Omega_{c}^{n}(M)$ with support in a set $K$; by using a partition of unity we may without limitation of generality assume that $K$ is contained a chart domain $U$. For small $\varepsilon, \operatorname{supp} \Phi_{\varepsilon, x} \subseteq U$ for all $x \in \operatorname{supp} \omega$, thus $\left\langle\left\langle u, \mathrm{~L}_{X_{1}}^{x} \ldots \mathrm{L}_{X_{k}}^{x} \Phi_{\varepsilon, x}\right\rangle, \omega(x)\right\rangle$ equals $\left\langle\left\langle u_{U}, \mathrm{~L}_{\left(X_{1}\right)_{U}}^{x} \ldots \mathrm{L}_{\left(X_{k}\right)_{U}}^{x}\left(\Phi_{U}\right)_{\varepsilon, x}\right\rangle, \omega_{U}(x)\right\rangle$ and converges to $\left\langle\mathrm{L}_{\left(X_{1}\right)_{U}} \ldots \mathrm{L}_{\left(X_{k}\right)_{U}} u_{U}, \omega_{U}\right\rangle$ which in turn equals $\left\langle\mathrm{L}_{X_{1}} \ldots \mathrm{L}_{X_{k}} u, \omega\right\rangle$.

(SK6): Fixing $K \subset \subset M$ for verifying (SK1) - (SK3) for $\mu^{*} \Phi^{\prime}$, we may assume that there are charts $(U, \varphi)$ on $M$ and $\left(U^{\prime}, \varphi^{\prime}\right)$ on $M^{\prime}$ such that $K \subset \subset U$ and $\mu(U)=U^{\prime}$. Given $\Phi^{\prime} \in \widetilde{\mathcal{A}}_{q}\left(M^{\prime}\right)$ there exists, by (SK4), a smoothing kernel $\Psi^{\prime} \in \widetilde{\mathcal{A}}_{q}\left(U^{\prime}\right)$ such that $\Phi_{\varepsilon, x}^{\prime}=\Psi_{\varepsilon, x}^{\prime}$ for $x \in \mu(K)$ and small $\varepsilon$, to which by Proposition 30 there corresponds a local smoothing kernel $\tilde{\psi}^{\prime} \in \widetilde{\mathcal{A}}_{q}\left(\varphi^{\prime}\left(U^{\prime}\right)\right)$. The diffeomorphism $\mu^{\prime}:=\varphi^{\prime} \circ \mu \circ \varphi^{-1}$ from $\varphi(U)$ to $\varphi^{\prime}\left(U^{\prime}\right)$ gives, by (LSK6), a local smoothing kernel $\tilde{\phi}:=\mu^{\prime *} \tilde{\psi}^{\prime} \in \widetilde{\mathcal{A}}_{q}(\varphi(U))$ to which in turn there corresponds a smoothing kernel $\Phi \in \widetilde{\mathcal{A}}_{q}(U)$. Because $\left(\mu^{*} \Psi^{\prime}\right)_{\varepsilon, x}=\Phi_{\varepsilon, x}$, the result is obtained. 
(LSK7) has no direct equivalent on the manifold. We come to the definition of moderateness and negligibility.

Definition 33 (D3, D4). (i) $R \in \hat{\mathcal{E}}(M)$ is called moderate if $\forall K \subset \subset M$ $\forall j \in \mathbb{N}_{0} \exists q \in \mathbb{N}_{0} \exists N \in \mathbb{N} \forall \Phi \in \widetilde{\mathcal{A}}_{q}(M) \forall X_{1}, \ldots, X_{j}$ we have the estimate $\mathrm{L}_{X_{1}}^{x} \cdots \mathrm{L}_{X_{j}}^{x}\left(R\left(\Phi_{\varepsilon, x}, x\right)\right)=O\left(\varepsilon^{-N}\right)$ uniformly for $x \in K$. The set of all moderate elements of $\hat{\mathcal{E}}(M)$ is denoted by $\hat{\mathcal{E}}_{m}(M)$.

(ii) $R \in \hat{\mathcal{E}}(M)$ is called negligible if $\forall K \subset \subset M \forall j \in \mathbb{N}_{0} \forall m \in \mathbb{N} \exists q \in \mathbb{N}_{0}$ $\forall \Phi \in \widetilde{\mathcal{A}}_{q}(M) \forall X_{1}, \ldots, X_{j}$ we have the estimate $\mathrm{L}_{X_{1}}^{x} \cdots \mathrm{L}_{X_{j}}^{x}\left(R\left(\Phi_{\varepsilon, x}, x\right)\right)=$ $O\left(\varepsilon^{m}\right)$ uniformly for $x \in K$. The set of all negligible elements of $\hat{\mathcal{E}}(M)$ is denoted by $\hat{\mathcal{N}}(M)$.

Corollary 34. Let $(U, \varphi)$ be a chart on $M$. Then $R \in \hat{\mathcal{E}}(U)$ is moderate or negligible, respectively, if $R_{U} \in \hat{\mathcal{E}}(\varphi(U))$ is so.

Proof. Using the relation $R\left(\Phi_{\varepsilon, x}, x\right)=R_{U}\left(\left(\Phi_{U}\right)_{\varepsilon, \varphi x}, \varphi x\right)$ the claim is immediate from the definitions and Proposition 30.

Again we can get rid of the derivatives in the test for negligibility.

Corollary 35. $R \in \hat{\mathcal{E}}_{m}(M)$ is negligible if and only if Definition 33 (ii) holds for $j=0$, which is, $\forall K \subset \subset M \forall m \in \mathbb{N} \exists q \in \mathbb{N}_{0} \forall \Phi \in \widetilde{\mathcal{A}}_{q}(M) R\left(\Phi_{\varepsilon, x}, x\right)=O\left(\varepsilon^{m}\right)$ uniformly for $x \in K$.

Definition 36. Let $R \in \hat{\mathcal{E}}(M)$ and $M^{\prime} \subseteq M$ open. Then the restriction $\left.R\right|_{M^{\prime}} \in$ $\hat{\mathcal{E}}\left(M^{\prime}\right)$ is defined as $\left.R\right|_{M^{\prime}}(\omega, x):=R(\omega, x)$ for $\omega \in \Omega_{c}^{n}\left(M^{\prime}\right) \subseteq \Omega_{c}^{n}(M)$ and $x \in M^{\prime}$.

As in the local case the following is an immediate consequence of (SK4).

Proposition 37. (i) Let $M^{\prime} \subseteq M$ be open and $R \in \hat{\mathcal{E}}(M)$. If $R$ is moderate or negligible, respectively, then so is $\left.R\right|_{M^{\prime}}$.

(ii) Let $\left(U_{\alpha}\right)_{\alpha}$ be an open covering of $M$ and $R \in \hat{\mathcal{E}}(M)$. If for all $\alpha,\left.R\right|_{U_{\alpha}}$ is moderate or negligible, respectively, then so is $R$.

By Proposition 37 (i) restriction is well-defined also on the quotient space:

Definition 38. Let $\hat{T} \in \hat{\mathcal{G}}(M)$ and $M^{\prime} \subseteq M$. Then the restriction $\left.\hat{T}\right|_{M^{\prime}} \in$ $\hat{\mathcal{G}}\left(M^{\prime}\right)$ of $\hat{T}$ to $M^{\prime}$ is defined as $\left.\hat{T}\right|_{M^{\prime}}:=\left.T\right|_{M^{\prime}}+\hat{\mathcal{N}}\left(M^{\prime}\right)$ where $T \in \hat{\mathcal{E}}_{m}(M)$ is any representative of $\hat{T}$.

Proposition 39. $\hat{\mathcal{G}}$ is a fine sheaf.

Proof. The proof of Proposition 18 applies with the obvious modifications; additionally, $\hat{\mathcal{G}}$ is fine because it is locally fine $([\underline{6]})$.

Theorem 40. (i) $\iota\left(\mathcal{D}^{\prime}(M)\right) \subseteq \hat{\mathcal{E}}_{m}(M)$, (ii) $\sigma\left(C^{\infty}(M)\right) \subseteq \hat{\mathcal{E}}_{m}(M)$, (iii) $(\iota-$ $\sigma)\left(C^{\infty}(M)\right) \subseteq \hat{\mathcal{N}}(M)$, (iv) $\iota\left(\mathcal{D}^{\prime}(M)\right) \cap \hat{\mathcal{N}}(M)=\{0\}$.

Proof. Insteaf of proving this directly we use the local results: for (i), $\iota u$ is moderate if $\left.\iota u\right|_{U}=\iota\left(\left.u\right|_{U}\right)=\left(\iota\left(u_{U}\right)\right)^{U}$ is so on each chart domain $U$, which by Corollary 34 is the case because $\iota\left(u_{U}\right)$ is moderate; similarily for (ii) and (iii). For (iv), $\left.\iota u\right|_{U}$ and thus $\iota\left(u_{U}\right)$ are negligible, which implies $u_{U}=0$ for all chart domains $U$ and thus $u=0$. 
$\hat{\mathcal{E}}_{m}(M)$ is a subalgebra of $\hat{\mathcal{E}}(M)$ and $\hat{\mathcal{N}}(M)$ is an ideal in $\hat{\mathcal{E}}_{m}(M)$, so we can define the algebra of generalized functions on $M$ as the quotient of moderate modulo negligible functions.

Definition 41. $\hat{\mathcal{G}}(M):=\hat{\mathcal{E}}_{m}(M) / \hat{\mathcal{N}}(M)$.

Theorem 42. $\hat{\mathrm{L}}_{X}$ preserves moderateness and negligibility.

Proof. Once again using (LSK4) one sees that $\left.\left(\hat{\mathrm{L}}_{X} R\right)\right|_{U}$ is moderate or negligible, respectively, if and only if $\left.\hat{\mathrm{L}}_{\left.X\right|_{U}} R\right|_{U}$ is so for all chart domains $U$, which by Corollary 34 is the case if and only if $\left(\left.\hat{\mathrm{L}}_{\left.X\right|_{U}} R\right|_{U}\right)_{U}=\hat{\mathrm{L}}_{X_{U}} R_{U}$ is moderate or negligible, respectively, which holds by Theorem 19.

Definition 43. $R, S \in \hat{\mathcal{E}}_{m}(M)$ are called associated with each other, written $R \approx S$, if $\forall \omega \in \Omega_{c}^{n}(M) \exists q \in \mathbb{N} \forall \Phi \in \widetilde{\mathcal{A}}_{q}(M): \lim _{\varepsilon \rightarrow 0} \int(R-S)\left(\Phi_{\varepsilon, x}, x\right) \omega(x)=0$.

This definition is independent of the representatives and extends to $\hat{\mathcal{G}}(M)$ because elements of $\hat{\mathcal{N}}(M)$ are associated with 0 . The notion of association localizes as well:

Lemma 44. (i) Given $R, S \in \hat{\mathcal{E}}_{m}(M)$ and an open cover $M, R \approx S$ if and only if $\left.\left.R\right|_{U} \approx S\right|_{U}$ for all sets $U$ of the cover. In particular, $R \approx S$ implies $\left.\left.R\right|_{U} \approx S\right|_{U}$ for any open subset $U$ of $M$.

(ii) Given $R, S \in \hat{\mathcal{E}}_{m}(U)$ for a chart domain $U, R \approx S$ if and only if $R_{U} \approx S_{U}$.

Proof. (i) is proven exactly as Lemma 22 while (ii) follows immediately from the definitions.

As before, we have:

Proposition 45. (i) For $f \in C^{\infty}(\Omega)$ and $u \in \mathcal{D}^{\prime}(\Omega), \iota(f) \iota(u) \approx \iota(f u)$.

(ii) For $f, g \in C(\Omega), \iota(f) \iota(g) \approx \iota(f g)$.

Proof. (i) $\iota(f) \iota(u) \approx \iota(f u)$ if and only if $\left.\left.\left.\iota(f)\right|_{U} \iota(u)\right|_{U} \approx \iota(f u)\right|_{U}$ for all $U$ of an atlas, which is the case if and only if $\iota\left(f_{U}\right) \iota\left(u_{U}\right) \approx \iota\left((f u)_{U}\right)$; and similarly for (ii).

This work was supported by projects P20525 and P23714 of the Austrian Science Fund (FWF).

\section{References}

[1] G. E. Bredon. Sheaf theory. Springer Verlag, New York, second edition, 1997.

[2] J. F. Colombeau and A. Meril. Generalized functions and multiplication of distributions on $\mathcal{C}^{\infty}$ manifolds. J. Math. Anal. Appl., 186(2):357-364, 1994.

[3] J. F. Colombeau. New Generalized Functions and Multiplication of Distributions. Elsevier Science Publishers B.V., Amsterdam, 1984. 
[4] J. F. Colombeau. Elementary introduction to new generalized functions. Elsevier Science Publishers B.V., Amsterdam, 1985.

[5] G. M. Constantine and T.H. Savits. A multivariate Faa di Bruno formula with applications. Trans. Am. Math. Soc., 348(2):503-520, 1996.

[6] C. H. Dowker. Lectures on sheaf theory. Tata Institute of Fundamental Research, Bombay, 1956.

[7] M. Grosser, E. Farkas, M. Kunzinger, and R. Steinbauer. On the foundations of nonlinear generalized functions I and II. Mem. Amer. Math. Soc., 153(729), 2001.

[8] M. Grosser, M. Kunzinger, M. Oberguggenberger, and R. Steinbauer. Geometric Theory of Generalized Functions with Applications to General Relativity. Kluwer Academic Publishers, Dordrecht, 2001.

[9] M. Grosser, M. Kunzinger, R. Steinbauer, and J. A. Vickers. A global theory of algebras of generalized functions. Adv. Math., 166(1):50-72, 2002 .

[10] M. Hardy. Combinatorics of partial derivatives. Electron. J. Combin., 13(1), 2006.

[11] J. Jelínek. An intrinsic definition of the Colombeau generalized functions. Commentat. Math. Univ. Carol., 40(1):71-95, 1999.

[12] J. Jelínek. Equality of two diffeomorphism invariant Colombeau algebras. Commentat. Math. Univ. Carol., 45(4):633-662, 2004.

[13] A. Kriegl and P. Michor. The convenient setting of global analysis, volume 53 of Mathematical Surveys and Monographs. American Mathematical Society, Providence, RI, 1997.

[14] M. Kunzinger and E. A. Nigsch. Manifold-valued generalized functions in full Colombeau spaces. Commentat. Math. Univ. Carol., 52(4):519-534, 2011.

[15] S. Mazur and W. Orlicz. Grundlegende Eigenschaften der polynomischen Operationen. Stud. Math., 5:50-68, 1935.

[16] E. A. Nigsch. Point value characterizations and related results in the full Colombeau algebras $\mathcal{G}^{e}(\Omega)$ and $\mathcal{G}^{d}(\Omega)$. Math. Nachr., 2012. To appear.

[17] M. Oberguggenberger. Multiplication of Distributions and Applications to Partial Differential Equations, volume 259 of Pitman Research Notes in Mathematics. Longman, Harlow, U.K., 1992.

[18] L. Schwartz. Sur l'impossibilité de la multiplication des distributions. Comptes Rendus de L'Académie des Sciences, 239:847-848, 1954. 